\title{
Scheduling and Delivering Aircraft to Departure Fixes in the NY Metroplex with Controller-Managed Spacing Tools
}

\author{
Eric Chevalley, ${ }^{1}$ Bonny Parke, ${ }^{2}$ Josh M. Kraut, ${ }^{1}$ Nancy Bienert, ${ }^{3}$ Faisal Omar ${ }^{2}$ \\ San Jose State University Foundation / NASA Ames Research Center, Moffett Field, CA, 94035 \\ and \\ Everett A. Palmer ${ }^{4}$ \\ NASA Ames Research Center, Moffett Field, CA, 94035
}

In this paper, successful Time-Based Flow Management (TBFM) scheduling systems for arrivals are considered and adapted to apply to departures. We present a concept of operations that integrates Controller-Managed Spacing tools for departures (CMS-D) with existing tactical departure scheduling tools to coordinate demand at departure fixes in a metroplex environment. We tested our concept in a Human-in-the-Loop simulation and compared the effect of two scheduling conditions: 1) "Departure Scheduling" consisting of an emulation of the Integrated Departure and Arrival Capability (IDAC) where Towers and a Planner (Traffic Management Coordinator at the appropriate facility) coordinate aircraft scheduled takeoff times to departure fixes; and 2) "Arrival Sensitive Departure Scheduling" where, in addition, the Tower and Planner also consider arrival Scheduled Time of Arrivals (STAs) at the airport's dependent runway. Results indicate little difference between the two scheduling conditions, but a large difference between the No Tools and the two scheduling conditions with CMS-D tools. The scheduling/CMS-D tools conditions markedly reduced heading, speed clearances, and workload for controllers who were merging flows at the departure fixes. In the tool conditions, departure controllers conditioned departures earlier rather than later when aircraft were tied near the departure fixes. In the scheduling/CMS-D tools conditions, departures crossed the departure fixes 50 seconds earlier and with an $8 \%$ error rate (consisting of time ahead or behind desired time of arrival) compared to a $19 \%$ error rate in the No Tool condition. Two exploratory runs showed that similar beneficial effects can be obtained only with the CMS-D tools without scheduling takeoff times, but at the cost of a somewhat higher workload for controllers, indicating the benefits of predeparture scheduling of aircraft with minimal delays. Hence, we found that CMS-D tools were very beneficial in the metroplex environment we tested but that further research is needed to clarify the benefits of the various scheduling approaches.

\section{Nomenclature}

$\begin{array}{ll}\text { ATC } & =\text { Air Traffic Control } \\ \text { CFR } & =\text { Call For Release } \\ \text { CLT } & =\text { Charlotte airport } \\ \text { CMS } & =\text { Controller-Managed Spacing } \\ \text { CMS-D } & =\text { Controller-Managed Spacing for Departures } \\ \text { DCA } & =\text { Reagan National airport } \\ \text { DSP } & =\text { Departure Sequencing Program } \\ \text { EDC } & =\text { En route Departure Capability } \\ \text { EDP } & =\text { Expedite Departure Path }\end{array}$

${ }^{1}$ Senior Research Psychologist, Human Systems Integration Division, NASA Ames Research Center, MS 262-4.

${ }^{2}$ Senior Research Associate, Human Systems Integration Division, NASA Ames Research Center, MS 262-4.

${ }^{3}$ Project Associate, Human Systems Integration Division, NASA Ames Research Center, MS 262-4.

${ }^{4}$ Human Factors Engineer, Human Systems Integration Division, NASA Ames Research Center, MS 262-4. 


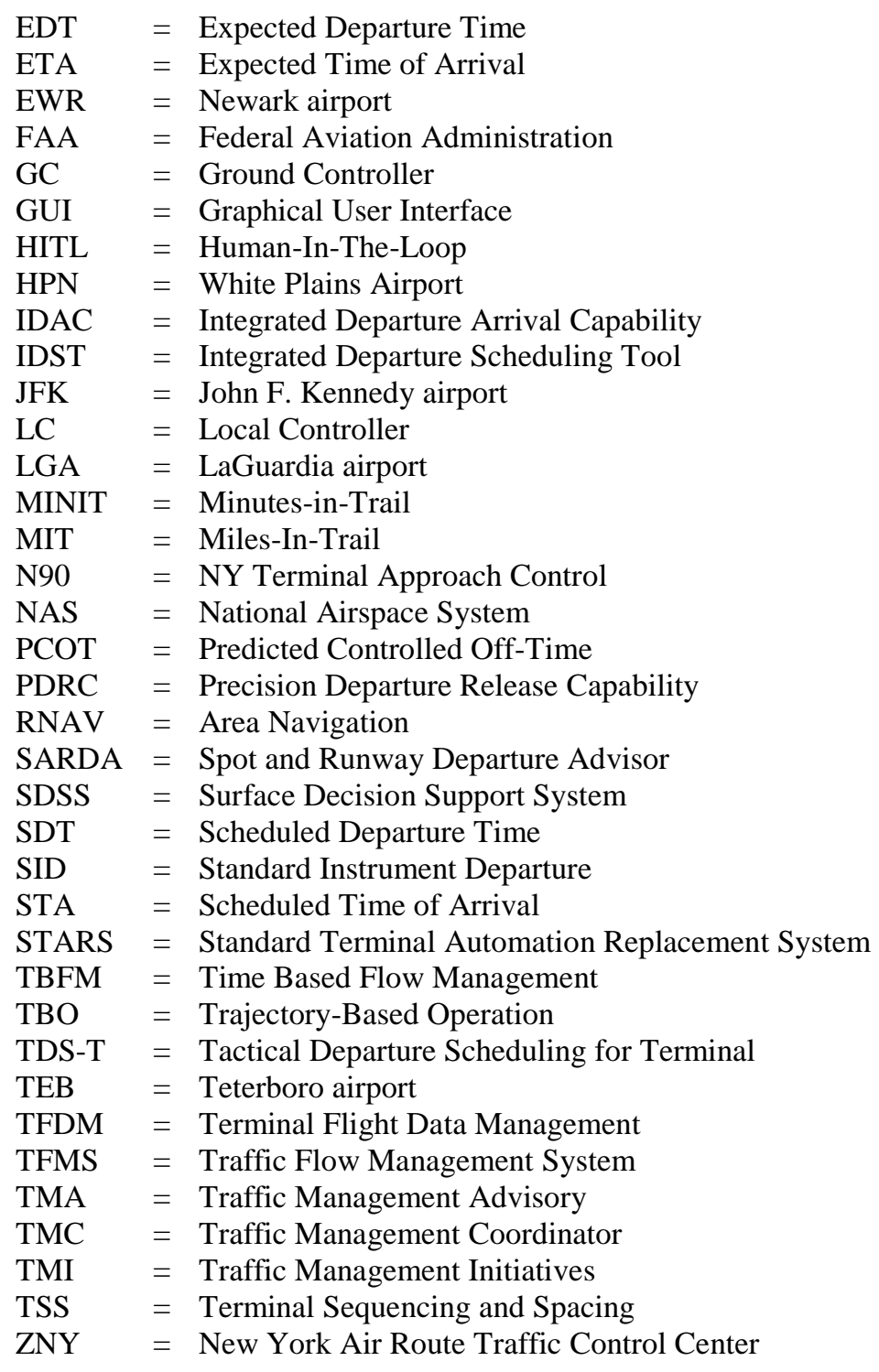

\section{Introduction}

M any of the delays in the National Airspace System (NAS) today are due to the lack of predictability in high density operations. This often leads to congestion and increased controller and pilot workload. Congestion in traffic flows due to uncoordinated demand is a common problem both for arrival and departure operations. This leads to inefficiencies, such as wasted airspace capacity, and suboptimal throughput and trajectories.

Improving traffic flow management has become one of the main goals of the Federal Aviation Administration (FAA). Since 2003 the Joint Planning and Development Office (JPDO) and the FAA have worked on a Next Generation (NextGen) Air Transportation System Implementation Plan to modernize technologies and procedures and improve efficiency across the NAS. ${ }^{1}$ One of the FAA's long term objectives is to develop Gate-to-Gate scheduling. This means controlling the time of flights at pushback from the gate, at takeoff time, at the departure fix, at the arrival meter fix, at touchdown, and at the arrival gate. Gate-to-Gate scheduling would require the integration of three major software systems: Traffic Flow Management System (TFMS), Time-Based Flow Management (TBFM) and Terminal Flight Data Management (TFDM). For instance, the airline pushback time would need to be coordinated with the tactical takeoff time (TFDM-TBFM). Strategic Traffic Management Initiatives (TMIs) and arrival metering would need to be coordinated (TFMS-TBFM). TBFM is thus a critical component of Gate-to-Gate scheduling. It provides the technology to potentially integrate departure flows with arrival flows. 
However, today there is a large technology gap with departure flow management. TBFM solutions have mostly supported arrival traffic flow management problems (Section A). Departure solutions have emerged to better estimate and manage take-off times that are restricted by Traffic Management Initiatives (TMIs) (Section B). However, further development is needed to include the flow management from multiple airports as well as to provide tools for controllers to achieve required scheduled times and optimal throughput during the climb-out phase.
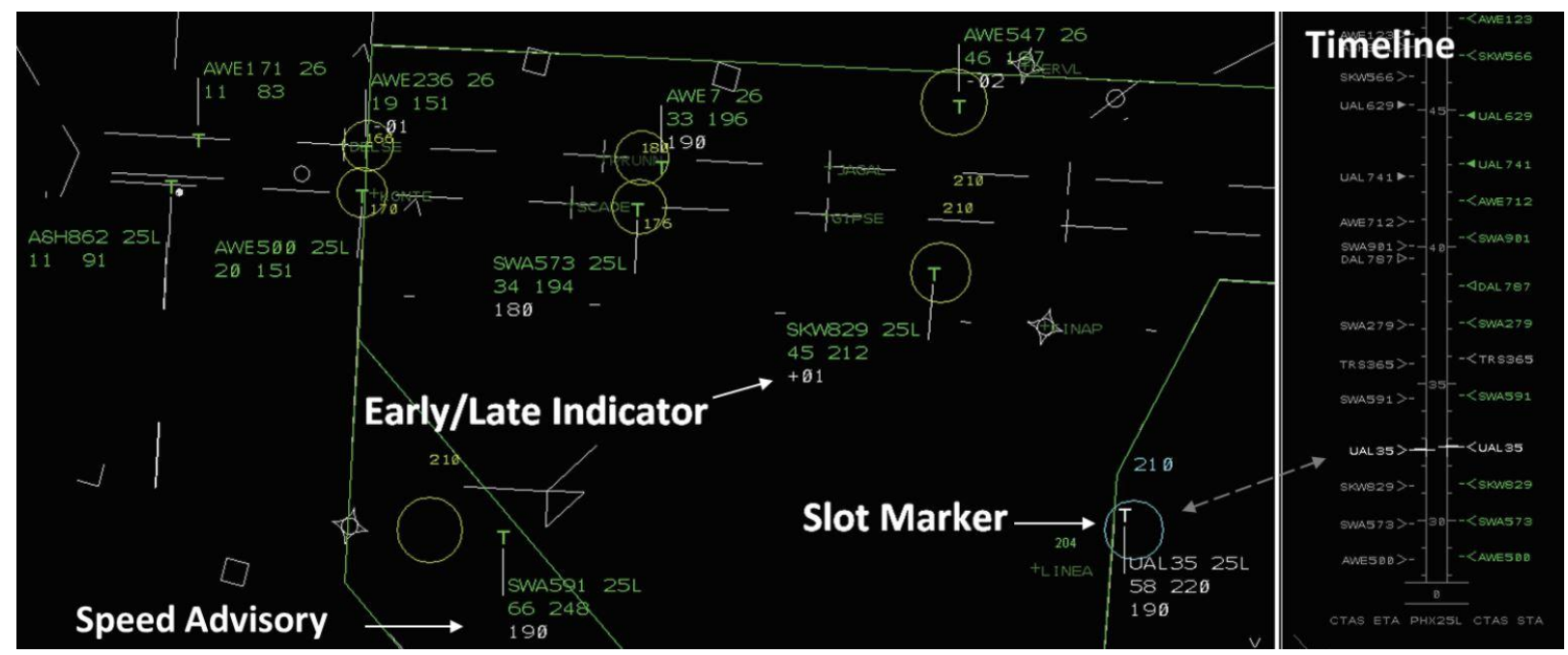

Figure 1. Controller Managed Spacing tools.

\section{A. TBFM Solutions for Arrival Scheduling Problems}

In the past decade, NASA Ames has collaborated with the FAA to develop the Traffic Management Advisor (TMA) technology under the currently fielded TBFM system. Terminal Sequencing and Spacing (TSS), the most recent NASA technology, has been transferred to the FAA and soon will also be part of new Air Traffic Control (ATC) capabilities. TSS scheduling automation relies on trajectory prediction along Area Navigation (RNAV) routes, and will enhance TMA's scheduling capability by de-conflicting arrivals at meter points and merge points all the way to the runway threshold. Additionally, TSS is coupled with the Controller-Managed Spacing (CMS) tool set. $^{2,3}$ The CMS tools support terminal controllers in achieving the scheduled plan by portraying three sets of information on the controllers' Standard Terminal Automation Replacement System (STARS) screen, as shown in Fig. 1:

1) A timeline displays the Expected Times of Arrival (ETAs) and the Scheduled Times of Arrival (STAs) of each aircraft at a fix point, usually a merge point. The difference between the ETA and STA indicates whether the aircraft is early or late. Early/Late indicator information is also displayed below the aircraft's datablock.

2) Slot marker circles indicate the scheduled spatial location of aircraft on the STARS display. These circles show where aircraft should be to meet their intended schedules, given their planned trajectory and any restrictions. They provide a visual reference of the aircraft's current and desired location.

3) A speed advisory shows recommended airspeed for aircraft to meet their STAs.

All of the computations for the aircraft ETAs and STAs take into account the current and planned flight trajectory as well as forecast winds.

TSS has been shown to increase traffic flow management efficiency and throughput during high-volume traffic. Additionally, this type of Trajectory-Based Operations (TBO) combined with CMS tools increases the predictability and controllability of aircraft. These capabilities help reduce delays and increase aircraft schedule conformance. Lastly, delay absorption is distributed across controllers and sectors, and as a result, reduces controller workload. ${ }^{2-4}$

\section{B. Departure Flow Management Scheduling Problems}

Integrating departures into en route traffic can cause three kinds of scheduling problems for en route Traffic Management Coordinators - two outbound and one inbound. ${ }^{5-7}$ These problems are:

1) Adjusting the volume of outgoing departures entering a sector given the sector and departure fix capacity,

2) Inserting outgoing departures into gaps in overhead traffic flows, 
3) Balancing incoming departures to an arrival meter fix to a nearby destination airport (e.g. "internal departures").

These problems are often aggravated by flow restrictions (Miles-in-Trail), usually imposed by adjacent en route facilities onto the facility that manages the departures. For these reasons, en route facilities require coordinated tactical departure scheduling with Towers. Call For Release (CFR) procedures, also known as Approval Request (APREQ) procedures, are put in place to manage the release of departures. These procedures require the Tower to request (usually by telephone) that the en route facility provide a takeoff time, which typically is bounded by a three minute window ( 2 minutes early, 1 minute late). CFR procedures are necessary to manage the flow of departures, but remain inefficient today.

Challenges: The success of managing departure flows is dependent on the management of the demand schedule, the compliance to the schedule, and the predictability and efficiency of the CFR scheduling procedure. The following departure flow management challenges are discussed below:

1) Management of schedule and flow restrictions,

2) Flight time predictability and compliance,

3) Takeoff predictability and compliance,

4) Coordination of release times,

5) Coordination of departures from multiple airports.

\section{Management of schedule and flow restrictions}

One of the challenges for the en route TMCs is to manage demand over restricted fixes. This includes fixes within the terminal, but mostly involves fixes at the en route facility boundary. Commonly, en route TMCs manage excess demand by placing Miles-In-Trail (MIT) flow restrictions to departure fixes. ${ }^{7-9}$ These restrictions are one of the TMIs available for traffic management. MIT are often imposed by downstream en route centers which apply restrictions at their boundary to upstream centers to manage the demand in their own sectors. This practice is called "passback MIT". Unfortunately, this practice does not take into account fluctuations of the demand in the upstream sectors and thus can create unnecessary compression on the traffic. If the en route facility has delegated some of its airspace to a terminal facility, then these restrictions are often imposed on the terminal sectors that feed the en route sectors. Sometimes, a buffer will be added to the MIT. MIT restrictions onto terminal sectors are used to give en route controllers either the rate of traffic they can manage or to provide them with adequate spacing for merging departures with overhead traffic. MIT are driving the schedules used by the en route TMC. However MIT restrictions are not precise in adjusting to actual traffic flow, and can overly constrain the demand.

Today TMCs can use a more flexible TBFM capability called En route Departure Capability (EDC) to schedule departures. ${ }^{5,6}$ The en route facility that schedules departures into its own en route streams will try to meet the restrictions imposed by adjacent facilities. Unless an adjacent center is scheduling these departures into its own scheduling stream, the two schedules will be decoupled across the facilities.

EDC's extended metering and coupled scheduling functions are addressing the problem of integrating flows across facilities. These functions can meter arrivals up to about $700 \mathrm{~nm}$ from the airport. ${ }^{5,10}$ In theory these scheduling functions should facilitate the control of departure flows into arrival flows. Coupled Scheduling indicates that two schedules are inter-connected. However, in reality, the first scheduler is set to condition flows into the second scheduler independently without creating excessive delays.

The integration of independent schedules and local constraints remains a challenge.

\section{Flight time predictability and compliance}

Before TBFM departure capabilities such as EDC were deployed in the field, TMCs would calculate the runway departure time by manually subtracting the flight time to a fix from the desired time for the departure to be at the fix. For instance, if a departure needed to cross a fix at 12:15, the TMC would deduct the flight time of 00:15 to the fix, and calculate the release time to be 12:00. Conversations with Subject Matter Experts (SMEs) suggest that, at best, TMCs with experience would adjust this time based on the aircraft type, the airline, and the weather. Skilled TMCs would also take into account the traffic and the ATC personnel and create a slight compression over the fix to maintain throughput. However, this approach is not reliable due to the obvious difficulty of considering all factors at once, including airline-specific procedures related to bank angles and engine settings. Manually-calculated flight times at best are an approximation.

EDC automation has improved flight time estimation and thus the estimate of takeoff times. EDC estimates departure trajectories based on defined routes, weather forecast, and aircraft engine types. ${ }^{7}$ Provided that route definitions have been specified in the TBFM adaptation, EDC will then estimate when the departure should take off to meet the specified time. 
However, departure routes are frequently not specifically defined in departure airspace. To increase predictability, departure routes should be connected from the runway up to the schedule point in the en route airspace. The introduction of RNAV Standard Instrument Departure (SID) routes has helped to provide more certainty with lateral route trajectories. ${ }^{13}$ A remaining uncertainty is the climb trajectory of the aircraft, which may prevent the aircraft from meeting the predicted fight time. Thus, updated estimates of compliance are needed.

The prediction of flight time and the management of the trajectory were studied a decade ago with Expedite Departure Path (EDP). ${ }^{12-14}$ The goal of EDP was to support terminal controllers in sequencing, spacing, and merging departures into en route traffic flows. EDP used the trajectory synthesizer in the Center TRACON Automation System (CTAS, an older version of today's TBFM) to provide a conflict-free trajectory with heading and speed advisories for departure aircraft to be efficiently sequenced at a merge or departure fix. A route analyzer also computed a set of possible trajectories between the aircraft's current position and the departure fix. EDP enabled more efficient trajectories and better ETA precision. However, the accurate prediction of aircraft positions was shown to be difficult to achieve due to the variation of climb rates, aircraft weight, controller and pilot response delays, and winds.

Hence flight time compliance remains a problem, one that will be addressed in the current study.

\section{Takeoff time predictability and compliance}

The takeoff time is directly related to the management of surface movements. Without decision support tools, Ground Controllers (GCs) also manually calculate how long it will take for an aircraft to reach the runway and take off. A CFR procedure starts once the aircraft is in contact with GC, which sometimes takes place when the aircraft is still at the gate, and at other times, when the aircraft has left the ramp area and entered the Active Movement Area (AMA). The controller's prediction of when an aircraft can take off is influenced by the distance of the aircraft to the departure runway, the surface capacity (taxiways, spots, holding areas), and surface congestion (queues). The CFR procedure is usually not handled by the GCs or the local controllers (LCs) but by the tower TMC or other tower personnel. Unfortunately, the departure system currently in use (EDC) and its planned replacement (IDAC) lack the ability to estimate takeoff times from a surface data standpoint.

In recent years, NASA has developed two technologies to improve takeoff time predictability and compliance: Precision Departure Release Capability (PDRC) and Spot and Runway Departure Advisor (SARDA). These technologies support both surface and airborne operations, and hence support the integration of TFDM and TBFM. PDRC is a tactical departure scheduling tool that integrates both TMA and the Surface Decision Support System (SDSS, a TBO surface management system) to schedule off-times. By computing the Predicted Controlled OffTime (PCOT), which takes into account surface congestion, PDRC shows the earliest time an aircraft is likely to take off. This time is used by the automation to compute the assigned takeoff time for the en route TMC. ${ }^{5,15}$

The compliance with the current CFR takeoff-time window of 3 minutes may not deliver aircraft with enough precision to fit into existing slots in the en route airspace, especially when aircraft need to be inserted into a stream. A 3-minute window represents a distance of 18 nautical miles when departure speeds reaches 360 knots, which is the approximate ground speed at 20,000 feet. With this level of precision, controllers may still need to manipulate aircraft with speed or vectors, which results in inefficient trajectories, higher workload, and airborne delays. PDRC's field test at DFW showed a significant increase of takeoff time compliance with the 3-minute takeoff time-window from $54 \%$ in the baseline condition to $83 \%$ in the PDRC condition, with an error reduction of nearly a minute. ${ }^{5}$

Spot and Runway Display Aid (SARDA) is another NASA system that has been tested and shown to improve the reliability of takeoff times. SARDA uses time and trajectory-based operations on the surface to meet takeoff time restrictions. SARDA coordinates gate hold and pushback times with the ramp, meters departures at spots, and schedules optimal sequences of flows to the runway. Results show a reduction of surface delays, increased precision of takeoff times, and increased runway throughput. ${ }^{16,17}$

Another problem area relates to interdependent runway operations. Currently, the TBFM estimation of takeoff times does not take into consideration dependencies between arrival and departure runways. However, when arrival and departure operations are dependent, departures cannot take off until arrivals land. Dependent runway operations add uncertainty to the prediction of the actual takeoff times.

In the current study, we seek to increase the compliance of aircraft at the departure fix, and reduce takeoff time errors due to arrival and to departure runway dependencies. A later research question would be to assess whether the improvement of flight time and takeoff predictions could help reduce the need for restrictions at departure fixes. With more reliable departure ETAs at the departure fixes, there should be less wasted capacity and fewer airborne delays. More reliable ETAs at the departure fixes may also help assess the extent to which delays can be absorbed on the surface. 


\section{Coordination of release times across facilities}

The coordination of the takeoff times between the tower and the en route facilities requires processing and exchanging information. Today, while TMCs use EDC to compute takeoff times, CFR procedures are still accomplished verbally, which is time and labor intensive. These procedures also require two people to be on the phone at the same time which blocks the line for other requests. During high workload periods, the TMC may not be able to answer right away, further delaying the procedure. If a CFR procedure is in place, every single aircraft with a CFR needs to be processed in this way. Additionally, the TMC needs to maintain awareness of previous requests and expected future requests regarding available release times (slots) to assign. This awareness is difficult to maintain without a record of the requests, especially when they come from more than one airport. ${ }^{18}$

IDAC ${ }^{5}$ offers additional functions to EDC that allow electronic coordination between towers and the en route TMC, and which provide shared information across facilities. IDAC has a Graphical User Interface (GUI) called Integrated Departure Scheduling Tool (IDST). With IDST, a tower TMC can assign departures into takeoff time slots displayed on a runway timeline. The slots are back-propagated from the schedule point. The responsibility for authorizing the release time remains with the en route facility. Each tower sees only the slots that are available and cannot see scheduled departures from other towers. This is to prevent towers from competing with each other for slots. IDAC provides the en route TMCs with the option to automatically or semi-automatically accept the requested off-times by the towers. In sum, IDAC improves the coordination of the release time by eliminating verbal coordination and by providing more situational awareness of available times/slots across facilities. However, the tower still needs to manually assess when the departure is likely to take off. Without takeoff time predictions, slots can be missed and capacity can be wasted.

PDRC provides additional surface information that is shared across facilities. Both the en route TMC and the tower can see not only the runway timeline but also the surface delay. Based on this information, the TMC can assign a takeoff time that is more likely to be met. Coordination using PDRC starts with the tower sending an electronic request which shows up on the en route TMC display. The TMC initiates the scheduling and enters the desired time at the crossing fix. The automation then displays the PCOT based on surface information. The TMC decides on a takeoff time, adding delay if needed. This time is then electronically communicated to the tower to accept or reject. This provides a significant improvement in accuracy of predicted takeoff time compared to IDAC.

The remaining challenge in improving the reliability of the departure schedule is the integration of arrival STAs when departure and arrival runways are dependent, which we address in this study. We also combine the best features of IDAC and PDRC, such as allowing towers to schedule takeoff times and providing more accurate takeoff time predictions.

\section{Coordination of departures from multiple airports}

Metroplex environments add complexity to departure flow management since multiple airports release departures to shared departures fixes. Because of the competing demand across departure fixes, an additional challenge is the lack of predictability of the demand from the various airports. Various peaks of departure demand can create departure-fix congestion. For instance, in the NY metroplex, LGA departures peak in the morning and remain constant throughout the day, and EWR and JFK have departure pushes at different times of the day. This leads to uneven demand distribution across the departure fixes. There are also some difficulties with airports competing for departure release times. Airports furthest away may be able to get release times earlier than airports closer to the departure fix.

In metroplex airspace, separate terminal sectors frequently control departures from separate airports. Departure flows then converge into downstream en route sectors responsible for controlling these aircraft. Hence several controllers control departures until they reach their schedule point, without awareness of other constraints in other sectors. If takeoff times across airports are uncoordinated, controllers then end up controlling traffic that competes near or at departure fixes. At that point, controllers frequently need to delay traffic to separate aircraft and meet the required spacing at the departure fix. This creates inefficient trajectories and additional workload.

The coordination of departure releases provided by IDAC is expected to help de-conflict aircraft over the departure fixes and provide a flow that is better spaced. However, the en route TMC is still not aware of the predicted demand across airports. An extension of PDRC, called Tactical Departure Scheduling for Terminal (TDST), which includes the demand from multiple airports, is being developed to address that problem. ${ }^{21}$ Systems like TDS-T, PDRC and SARDA should help reduce the uncertainty of the takeoff time window. A remaining problem,

5 IDAC was derived from earlier prototypes such as Departure Flow Management (DFM) and Departure Release Communication. ${ }^{20,21}$ These prototypes also enabled non-verbal coordination. 
however, is the uncertainty of aircraft climb trajectories which will still impact the departures' actual time of arrival at the departure fixes.

The goal of this study is to emulate a metroplex problem and assess the efficiency of tactically scheduling departure takeoff times to improve the delivery of aircraft at the departure fixes with the help of CMS-D tools. In the next section, we present a concept of operations that we tested in a Human-In-The-Loop simulation. Results are then presented and discussed.

\section{Concept of Operations}

The concept of operations presented here leverages existing capabilities to address the remaining challenges.

First, a TBFM prototype scheduler, like TDS-T, is set at the terminal boundary of a metroplex environment. MIT restrictions are converted into time, and used as constraints to establish a schedule. A Metroplex Planner (TMC) manages the schedule of the demand at departure fixes. In this concept, the timelines show the schedules of all traffic, both overhead and terminal departures, that cross the departure fixes.

Second, RNAV departure routes start at the runway and connect all the way to the departure fixes. This allows for time-based operations, improved trajectory predictions (ETA), and more precise scheduling of departure aircraft. CMS-D tools are used to support terminal controllers in delivering departures to their STAs at the departures fixes.

Third, there is integration of both departure and arrival ETAs and STAs at the runway. Arrival STAs are used to more precisely schedule the departures takeoff times at airports with runway dependent operations. Our concept assumes optimal time-based operations on the surface.

Fourth, the concept integrates elements of PDRC and IDAC's capabilities. Both the tower coordinators (hereafter called "Towers"), and the Metroplex Planner both have runway timelines with predictions of takeoff times for departures. Towers can also initiate the scheduling by assigning departures into departure fix slots using their runway timelines. Advantages are 1) that runway slots are displayed to the Metroplex Planner and 2) that arrival STAs are displayed to the Towers that schedule departures.

Overall, the concept integrates the complementary capabilities of CMS-D tools with the scheduling functions of PDRC and IDAC. This integration of tools is particularly relevant in a metroplex environment prone to congestion with competing demands from various flows. The test of the concept as tested operationally in the simulation is described in more detail below.

\section{A. Prototype of Controller-Managed Spacing for Departures (CMS-D) as Tested in the HITL}

\section{Performance-Based Navigation (PBN)}

Arrival and departure routes in the terminal area are RNAV procedures. Arrival STARs procedures extend from the meter fix to the final approach fix. Arrival routes are Optimal Profile Descents. Departure SIDs start from the runway and connect to the departure fixes. SIDs have a few altitude restrictions to keep aircraft inside sectors and where procedural separation is needed (e.g. at merge points or when crossing traffic flows).

Route definitions are needed to assess flight time and to determine scheduled off-times. Route definitions are also needed to manage delays along known trajectories using the CMS-D tools.

\section{2. $C M S$ - $D$ for departures}

One novelty of this concept is the adaptation of the CMS tools used for arrivals to manage the departure climb out. With RNAV routes connecting runways to the departure fixes, it is possible to compute an ETA and STA to the departure fix. Departure takeoff times are scheduled based on aircraft's estimated time to the departure fixes. The terminal departure controllers use the CMS-D tools to absorb delays and to space departures at the departure fixes according to their STAs. Controllers can use both timelines and slot markers. Timelines display the Expected Times of Arrival (ETAs) and the Scheduled Times of Arrival (STAs) of each aircraft at the departure fix. The difference between the ETA and STA indicates whether the aircraft is early or late. Early/Late indicator information is also displayed below the aircraft's datablock. Slot markers are circles that indicate the scheduled spatial location of aircraft on the radar scope. These circles show where aircraft should be to meet their intended schedules, given their planned trajectory and any restrictions. They provide a visual reference of the aircraft's current and desired location.

\section{3. "TSS" (CMS) for arrivals}

Arrivals are de-conflicted and metered from the terminal meter fix down to the runway. Delays are managed by en route controllers to the meter fixes, and then terminal controllers use the CMS tools to absorb the remaining delay 
and deliver arrivals at the runway. On the terminal arrival sectors' scope, a timeline displays the ETAs and STAs of arrivals at the runway. Controllers also have slot markers as described above and in section I A.

\section{B. Elements of Time-Based Departure Scheduling (as Tested in the HITL)}

\section{Schedule points at terminal departure fixes}

Contrary to today's IDAC and EDC capabilities, the departure scheduling capability meters departures to departure fixes at the terminal boundary. Currently en route facilities typically schedule departures to downstream fixes that are restricted at the center's boundary. The goal is two-fold: 1) to provide departure flows that meet the required spacing or time; and 2) to deconflict traffic. The departure fixes can be considered as merge points given that MIT restrictions require in-trail spacing and that controllers tend to climb departures to a similar altitude range. (Although departures could be stacked vertically on top of each other if lateral spacing cannot be provided, controllers prefer to climb aircraft without restrictions and not be concerned with vertical clearances between two stacked aircraft.)

In this first evaluation of the concept, the Metroplex Planner schedules departures only to the departure fixes without overhead traffic. The goal, as described in the concept of operations, was to assess whether departures could be delivered to a schedule at the terminal boundary.

\section{Surface predictability of takeoff times}

The taxi-out time to the runway is predictable. Similar to SDSS technology as integrated in PDRC, an estimated takeoff time is provided on a runway timeline. Callsigns appear on the runway timeline after pushback as they are being tracked by SDSS. This information helps to calculate delays and feasible off-times. Departures were expected to take off as close to the scheduled time as possible.

\section{Flight time computation}

The computation of the takeoff time is dependent on the predicted flight time, which is computed by TBFM's route analyzer and trajectory synthesizer.

\section{Runway slots}

By metering both arrivals and departures, it is possible to predict scheduled takeoff slots during dependent runway operations. In such operations, controllers will tend to clear departure aircraft to take off after arrival aircraft have landed. This prevents loss of separation if the arrival aircraft needs to perform a missed approach after the departure is airborne.

\section{Metroplex TMC/Planner}

A metroplex TMC (the Planner) located at the TRACON facility is dedicated to managing the demand across the various departure fixes. The Planner makes sure the en route restrictions are met. The Planner sees the demand on a prototype Timeline Display as soon as aircraft heading for restricted departure fixes are tracked by the surface system.

\section{Information displayed on timelines}

Similar to IDAC's design, Towers can see slots on the runway timeline. These slots correspond to the available slots at the departure fixes, as shown in Fig. 2 (orange available slot marked with letter A). The delay between the runway and departure fix timeline is the average flight time, which provides a rough estimate of the location of any aircraft on either timeline.

On the departure fix timeline, the Planner can see the runway departure slots as well. The departure slots indicate which slots are available at the departure fix while taking into consideration the arrivals' STA at the runway as shown in Fig. 2 (example of blue available slot marked with letter I).

Similar to IDAC's IDST, the Tower sees only the aircraft that need a scheduled takeoff time in the timeline, as well as available slots at the departure fixes (Fig. 2). Slots occupied by departures from other airports are blacked out (Fig. 2 letter H). Similarly to PDRC, each aircraft on the timeline that is subject to a CFR is identified by having the letters "SCH" added to the callsigns (Fig. 2, letters A \& B). This indicates to the Tower that these aircraft need a scheduled off-time. In addition to "SCH", the first letter of the departure fix the flight is going to is indicated. This immediately informs the Tower which timeline to look for available slots.

Callsigns on the timelines can be color coded. On the Towers' timelines, callsigns are coded according to the departure fix they were flying to. On the Planner's timelines, the color matches the airport the departures come from. 
A departure list with callsigns and predicted takeoff times was also available to both the Tower and the Planner.

\section{CFR coordination}

The scheduling tool allows either the Tower or the Planner to assign a takeoff time first. In IDAC, the IDST permits the Tower to place departures into slots on a runway timeline. Then the en route TMC either accepts or rejects it. In PDRC, the Tower requests a time and the TMC can propose another departure time based on surface and departure fix information. The Tower then can accept or reject the time.

In the current concept, the coordination tool allows either party to initiate and accept a proposed scheduled time. Fig. 2 shows an example of possible coordination steps. LGA Tower initiates a coordination with the Metroplex Planner for flight TCF459. The Tower clicks on the "SCH" on the callsign's Expected Departure Time (EDT) on the runway timeline (A). A Scheduled Departure Time (SDT) then appears (B). The Tower then can move TCF459's callsign to the desired time (i.e., relative to a departure fix slot). The Tower then clicks on a "SUBMIT" button (B). TCF459's callsign is then backlit in white (C). This notifies the Tower that a request was sent. In the example, it can be seen that the slot that the Tower was requesting disappears before the time is accepted. On the Planner's end, the TCF492 callsign's STA appears on the departure fix timeline and is backlit in purple (E). This notifies the Planner that a response is expected for this callsign. The Planner then can accept, reject or modify the scheduled time. In this example, TCF492 conflicts with UAL872, another departure's STA that the Planner submitted to EWR airport. The Planner clicks on the TCF492's callsign. A menu showing "ACCEPT" or "REJECT" appears (E). In the example, the Planner has dragged TCF492's callsign to the next available slot (here 150s later). Now, a menu showing "SUBMIT", ACCEPT" or "REJECT" appears (F). The Planner has then clicked on "SUBMIT." The callsign is now backlit in white (F). The TCF492 callsign has moved on the Tower timeline and is backlit in purple, signaling that a response is needed (G). The Tower then clicks on "ACCEPT." TCF459's callsign gets frozen on both the Tower and Planner timelines ( $\mathrm{H} \& \mathrm{I})$. This completes the coordination cycle. When the Planner rejects a proposed time, the callsign's STA disappears, and on the Tower timeline the callsign displays the label "REJ". The Tower then has to start a new coordination cycle. A reschedule function unfreezes the callsign, which resets the process. In this case the Planner and the Tower need to coordinate verbally.

The example in Fig. 2 shows how conflicting demand is handled by the Planner. 


\section{Tower's view}

\section{Planner's view}
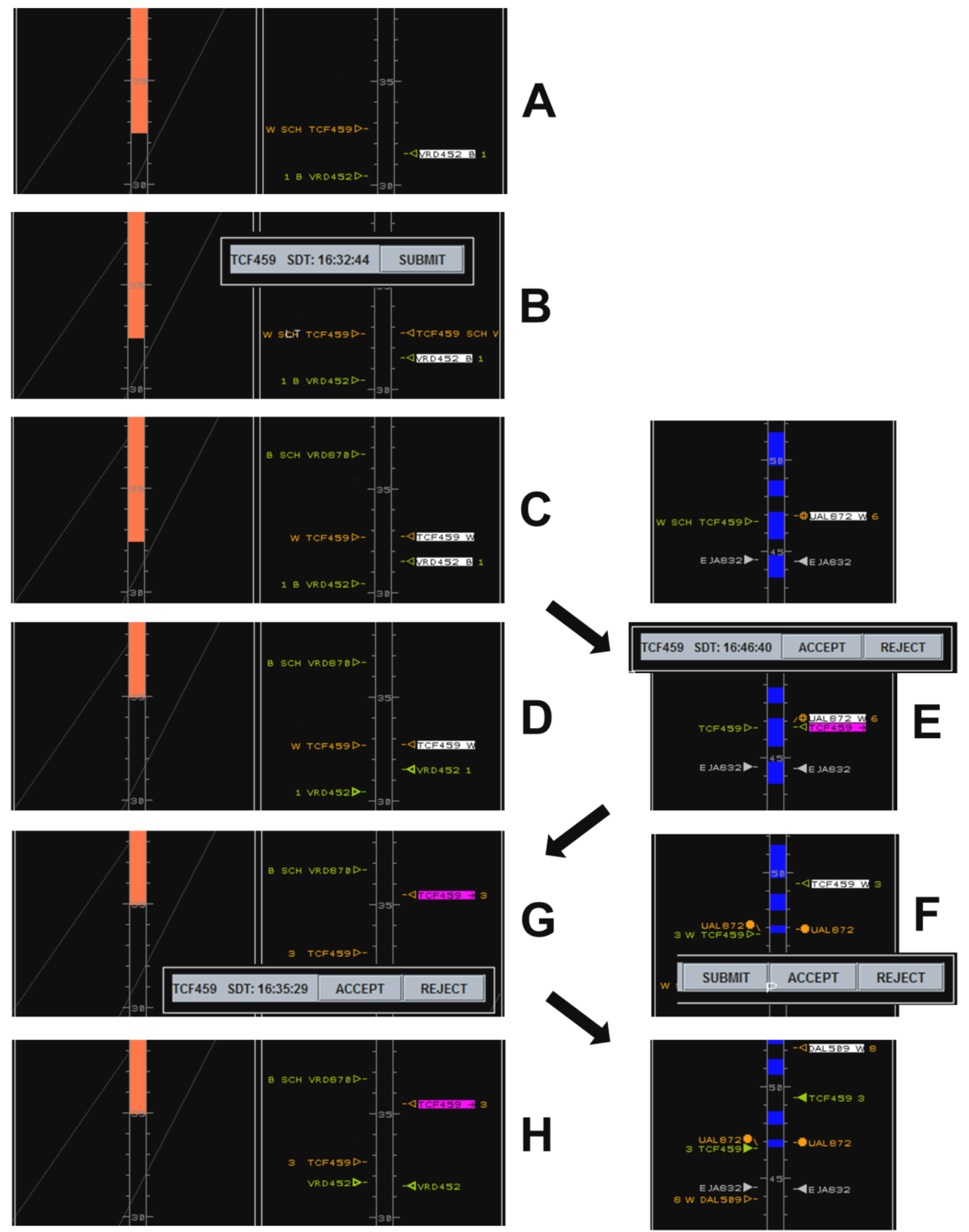

Figure 2. Example of coordination steps with the scheduling tool. 


\section{Concept Evaluation}

The above concept was tested in a Human-In-the-Loop (HITL) simulation at the Airspace Operations Laboratory at NASA Ames in 2014. The purpose of the simulation was to emulate the concept and compare it with current day operations using common metrics such as delay, schedule compliance and workload.

The method below describes the airspace and the scenario we emulated, as well as the experimental design.

\section{A. Simulated Metroplex Airspace}

The airspace that was simulated consisted of part of the departure airspace of the NY TRACON (N90) metroplex during a south flow configuration (Fig. 3). Some of that airspace was modified to allow for more optimal departure routes. Notably, the most southern portion of the EWR departure sector was widened from $5 \mathrm{~nm}$ to $20 \mathrm{~nm}$ to accommodate vectoring and more options to cross LGA arrival flows if the space was adequate.

NY departure terminal airspace is divided into two main layers. The first layers are the departure sectors that are above the main airports. EWR Departure controls aircraft departing from EWR and TEB. LGA Departure controls aircraft departing from LGA and HPN. JFK Departure controls

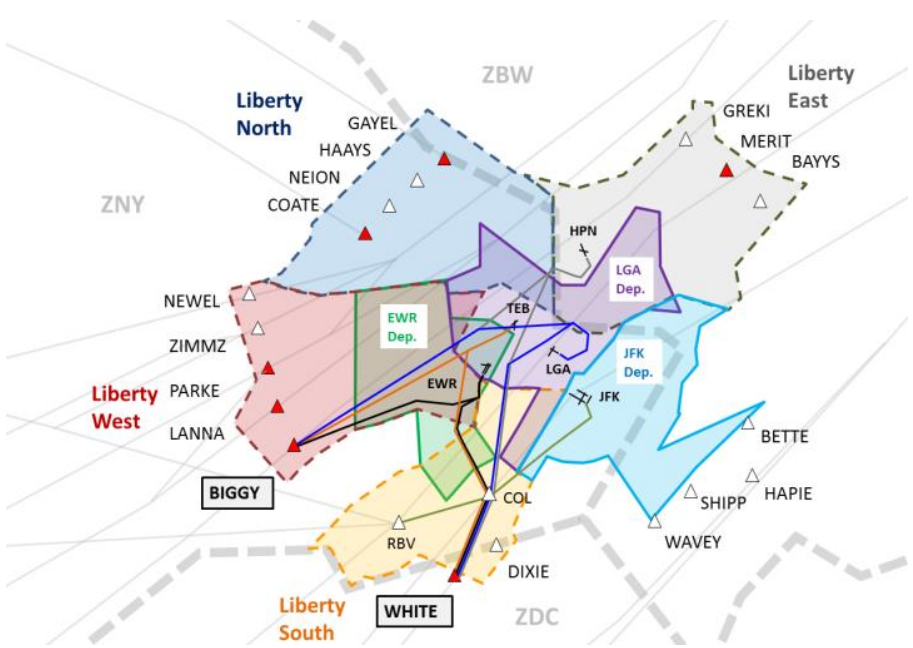

Figure 3. Simulated NY Departure Terminal Airspace.

aircraft departing from JFK. JFK Departure controls four departure fixes over the Atlantic which are mostly used by aircraft from JFK. With the exception of the JFK departure sector, EWR and LGA sectors control aircraft up to 10$12,000 \mathrm{ft}$.

Above the departure sectors are four Liberty sectors (also departure sectors). The Liberty sectors are divided according to the departure gates they control. In our simulation, these sectors control departures up to 22,000ft (instead of 17,000ft today). Liberty East controls the East Gate (GREKI, MERIT and BAYYS). Liberty North controls the North Gate (COATE, NEION, HAAYS and GAYEL). Liberty West controls the West Gate (BIGGY, LANNA, PARKE, ZIMMZ and NEWEL). Liberty West takes control of departures from both EWR and LGA departure sectors. Departures from JFK first fly through LGA Departure before they enter Liberty West. Liberty South controls the South Gate (WHITE and DIXIE). Liberty South takes control of departures from both EWR and LGA departure sectors. Some departures from JFK fly across the sector via RBV. In our simulation, traffic flew to all of the Liberty sectors, but only departures flying to BIGGY in Liberty West and to WHITE in Liberty South were evaluated.

\section{BIGGY and WHITE departures}

BIGGY and WHITE are among the departure fixes with the heaviest demand in NY, particularly during morning departure pushes. BIGGY is situated on Jetway 75. All the departures from EWR, LGA, TEB, and HPN that are going to BWI, CLT, DCA, plus fourteen other destinations, fly through BIGGY. In addition to that traffic, heavy JFK departure traffic flies through RBV before merging onto J75 after BIGGY. Enroute controllers are thus responsible for merging the JFK departures with the BIGGY departures and then with overhead traffic, and often restrict the NY departures for merging and capacity reasons.

WHITE is situated at the beginning of J209. J209 then merges with J79 at VILLS, near Atlantic City, where PHL departures fly. All the departures from EWR, LGA, TEB and HPN that are flying to FLL, MCO, and MIA, plus eleven other destinations, fly through WHITE. Additionally, the demand from TEB to WHITE will often increase during holiday seasons or during popular events. There is no merging-traffic problem at WHITE; the merging, if any, takes place further south with PHL departures. However, WHITE departures are often restricted for sector capacity reasons. The en route sectors were not included in the simulation, as they were beyond the scope of this first evaluation. Restrictions were assumed at the departure fixes.

In this simulation, departures flew from EWR, LGA, TEB, and HPN to BIGGY and WHITE. Liberty West controlled the BIGGY departures and Liberty South controlled the WHITE departures. The simulation emulated a 
departure push of more than 32 aircraft per hour over both departure fixes, during Visual Meteorological Conditions. The restrictions applied at the fixes were limited to 30 aircraft per hour, in other words, one aircraft every 2 minutes in the Tool condition, or 12 MIT in the No Tools condition (current day operations). This type of restriction provides enough space for overhead traffic during clear-weather conditions.

\section{Departure Sequencing Program}

Unlike anywhere else in the NAS, N90 departures are managed with a system called Departure Sequencing Program (DSP). It is used in ZNY, N90, and all 5 main airports within the terminal area (EWR, JFK, HPN, LGA \& TEB). DSP sets constraints on the sequence of departures from all airports it serves. ZNY uses DSP to control the rate of the demand at the departure fixes. The DSP flow rates are then translated into Minutes In Trail (MINIT) for all airports, except for JFK which receives MIT. EWR and LGA usually get the same MINITs for the same departure fixes. TEB and HPN also receive the same MINITs as each other. EWR and LGA have typically more demand at the departure fixes than TEB and HPN. For this reason the MINITs assigned to EWR and LGA are lower than those assigned to TEB and HPN.

Unfortunately, DSP does not know about the uneven demand across airports and therefore can issue disproportionate restrictions. For example, EWR may have three BIGGY departures in the next 15 minutes, and LGA may have six. Under DSP, LGA would need to delay three departures, instead of using the departure slots that EWR would not need.

DSP rates were used in the simulation. NY Subject Matter Experts (SMEs) recommended imposing the following restrictions to the airports to meet the rate of 30 aircraft an hour: 5MINIT for EWR and LGA, and 9MINIT to TEB and HPN.

\section{B. Assumptions}

We did not emulate surface operations to test the concept. Instead, we assumed that the towers' GC and LC were able to make departures comply with their takeoff times. Any delay at takeoff was because arrivals prevented departures from taking off on time or because there was a conflict of takeoff times between two departures with scheduled takeoff times. We used the first come first serve rule in such situations.

As in the PDRC suite of tools, a runway departure timeline was used to represent an aircraft's predicted takeoff time. When an aircraft appeared on the timeline, it meant that the aircraft had started moving onto the active surface movement area and would have been picked up by the surface system (SDSS). When aircraft reached the bottom of the timeline, they started rolling down the runway and took off.

RNAV departure routes were used in all the test conditions. Besides airspace modification, this was the only significant difference from current operations in the test airspace.

Traffic scenarios simulated a morning departure push over two departures fixes. We attempted to create a situation where demand was high enough to create compression and thus potential congestion. The demand at EWR and LGA were identical over the duration of the scenario. The terminal was assumed to be clear of weather and significant winds. Arrivals were scripted to enter the terminal airspace with $20 \mathrm{~s}$ or less delay and landed on time to the extent possible.

In this simulation, we did not distinguish whether the restrictions at the departure fixes were due to the need for stream insertion or for volume/capacity. The purpose was to require terminal controllers to deliver aircraft according to the MIT restriction or the schedule. This matches current practice in the field, regardless of its efficiency or lack thereof.

\section{Method}

1. Design

The experimental design consisted of three tool conditions that were repeated four times in a counter-balanced order. The three conditions were the following:

1) No Tools: The first condition reflected current-day operations in the NY Metroplex without any scheduling or delivery tools. In this condition, the releases of departures followed the DSP procedure. EWR and LGA departures were set to depart every 5 minutes and TEB and HPN every 9 minutes.

2) Spacing Tools + Departure Scheduling: In the second condition, the terminal controllers had the CMS-D tools for controlling departures. In addition, a Tower coordinator and a Metroplex Planner scheduled takeoff times with the help of the Tactical Departure Scheduling (TDS-T) capability described above. In this condition the Tower and the Metroplex Planner did not know about the arrivals' STA at the runway crossing. 
3) Spacing Tools + Arrival-Sensitive Departure Scheduling: In the third condition, in addition to the tools in the second condition, both the Tower and the Metroplex Planner were given information about the arrival STAs. The Tower could see the arrival STAs on the runway timeline. The Metroplex Planner could see the runway slots projected onto the departure fix timeline (depicted in blue in Fig. 2, letter E).

\section{Participants}

Seven retired controllers participated in the data collection. Two controllers had experience with tactical scheduling at towers. One controller had 30 years of experience as a controller and as a TMC at an en route facility. Four controllers had worked 15 years on average in a terminal facility and as a controller in all facilities for an average of 26 years. They had been retired for 4.3 years on average.

\section{Apparatus}

The simulation was run on the Multi-Aircraft Control System (MACS) software. MACS emulates STARS displays and are shown on large monitors similar to those used in the ATC facilities. Identical terminal keyboards and a Groundground and Air-ground voice communication system helped to replicate controllers' tools. Aircraft were piloted by pseudo-pilot stations. MACS provides a high fidelity environment to prototype time-based and trajectory-based technologies to simulate the air traffic and to collect data. ${ }^{22,23}$

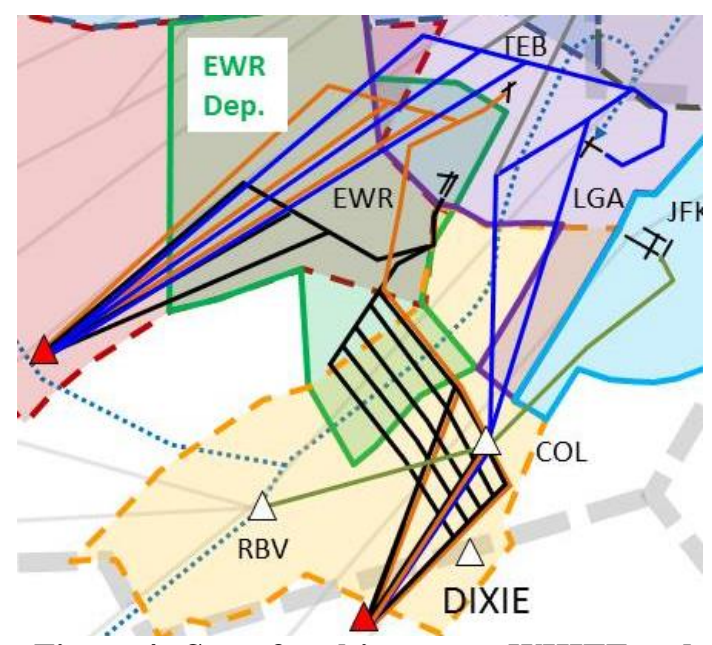

Figure 4. Sets of multiroutes to WHITE and to BIGGY.

\section{Other tools: Multiroute Options}

In this study, controllers used CMS-D tools along with a set of multiple RNAV routes (multiroutes). Multiroutes were composed of a nominal route, a shorter route, and a longer route (see the sets of routes in Fig. 4). A tool was prototyped to provide controllers with the option to change routes by clicking on the SID in the datablock (Fig. 5). A pop-up fly-out menu then appeared and indicated the predicted delay for each of the multiroute options. The tool computed the ETA at the departure fix and compared it to the STA, based on the current position of the aircraft, and for each route. Each route was treated as a possible known route, based on the RNAV procedure definition. The
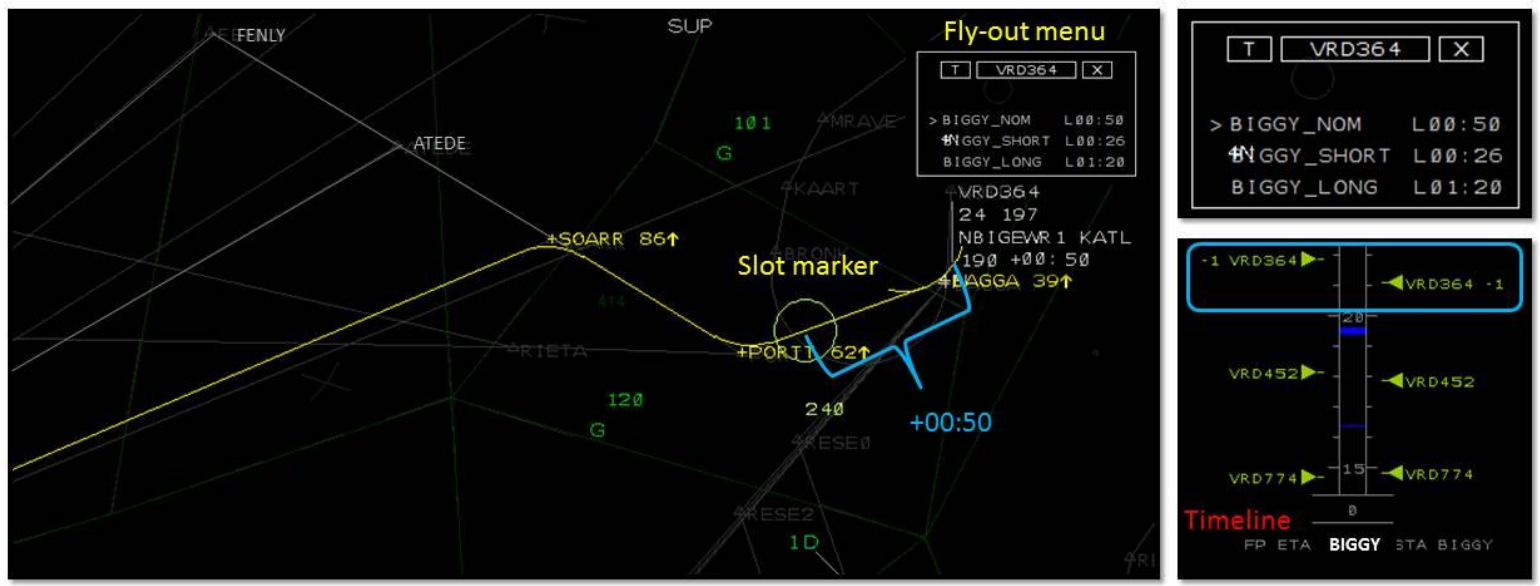

Figure 5. Example of multiroutes, fly-out menu, and timeline.

controllers could then click on a different route in the fly-out menu, which then updated the ground system. The controllers then communicated the new SID to the flight deck. In the study, the pilots were able to change the route rapidly. In the real world, such a rapid change of route might not be possible, depending on the aircraft type, and might require controllers to vector aircraft instead. 


\section{Scenarios}

Three different sets of scenarios were created. In each scenario, 40 aircraft took off from LGA, 37 from EWR, 25 from TEB, and 4 from HPN. About 35\% of departures flew to BIGGY, 35\% to WHITE and the remaining 30\% flew to other departure fixes. JFK departures did not fly to BIGGY and WHITE. However they were included in the scenario, as they fly across Liberty South towards RBV. The fleet mix was composed of small and large jet aircraft only. The mix of callsigns was based on real traffic flying out of the NY airports. Aircraft were randomly assigned weight factors to create a diversity of climb rates. Callsigns and their order were replaced automatically in each run. The departure demand from EWR, LGA, TEB and HPN was generated automatically by a script. Runs were unique, while keeping the traffic equal across conditions. The first two departures from each airport were already scripted to take off at the beginning of each run to create an existing demand and running operation.

The arrival rates were set at 40 aircraft an hour at LGA and EWR, and 20 at TEB.

\section{Tasks and procedures}

At the beginning of the runs, a script created expected departure times (EDTs) for the next two hours on the runway timelines. The Towers' timelines showed EDTs only for the next 30 minutes. A research confederate then populated the queue in the timeline by sequencing aircraft on a first come first served basis. This was done by updating the expected time and placing the callsign into the next available departure slot in the timeline. This time was considered as the Taxi Queue Entry Time.

As the BIGGY and WHITE aircraft started populating the Towers' timelines, the Tower TMCs then initiated the coordination of the scheduled off-time with the Metroplex Planner. The Planner and Towers were told that they should coordinate electronically up to two rejected times and then verbally coordinate. The Tower would send a proposed time, which would either be accepted or rejected by the Planner. If the proposed time was not accepted, the Planner would usually propose a new time. The new time was usually accepted by the Tower. A typical situation that led the Planner to reject or propose a new time was when two airports were competing over the same slot.

Once the scheduled times were set, the callsigns were frozen at the departure fix timeline, and were displayed as frozen on the runway timeline as well. This signaled the research confederate to adjust the expected off-time to the scheduled time. The confederate attempted to match the scheduled time as closely as possible. The decision was based on the following rules. 1) The expected departure time (EDT) needed to be right after or at least $45 \mathrm{~s}$ before an arrival ETA on the runway timeline. 2) The EDT could not overlap with another aircraft with a scheduled EDT. In this latter case, the EDT was placed to the next available departure slot. These rules were followed in all the tool conditions.

Controllers were required to meet the in-trail spacing of $12 \mathrm{~nm}$ in the No Tool condition and the STAs (separated by 120 s) in the tool conditions. Controllers were asked to let departures fly the RNAV SIDs, unless they had to vector aircraft for spacing or safety reasons.

\section{Results}

Metrics, such as average flight times, average delays (on the surface, at takeoff and at the departure fixes), number of clearances, as well as survey ratings were aggregated at a run level and tested for mean differences across conditions. In the figures shown, error bars are standard deviations. Descriptions and illustrations provide additional qualitative analyses.

\section{A. Scheduling Departures}

\section{Taxi-out delays}

We computed taxi-out delays by subtracting the unimpeded taxi-out time from the total taxi-out time used. (The unimpeded taxi-out times corresponded to the remaining time to EDT when the departure appeared on the runway timeline.)

Scheduling in the two tool conditions delayed departures only an average of 3.5 minutes $(M=3 \mathrm{~m} 31 \mathrm{~s}, S D=59 \mathrm{~s})$, $F(1,10)=2.4)$ compared to the No Tools condition of 5 minutes ( $40 \%$ larger) $(M=4 \mathrm{~m} 56 \mathrm{~s}, S D=2 \mathrm{~m} \mathrm{16s,} p=.15)$. In other words, metering with MINIT, in the No Tools condition created excess delay on the surface. This shows the advantage of scheduling based on departure fix demand rather than the constant restriction of MINIT at the runway. The larger surface delay in the No Tools condition is reflected by the number of CFR departures that took off. There were on average 4 fewer departures that took off in the No Tool condition $(M=56.2, S D=2.4)$ compared to the two tool conditions $(M=60.6, S D=2.1), F(1,10)=10.9, p=.008$. 
It is noteworthy that the lower taxi-out delays in the tool conditions is that LGA were an average of 1 minute less $(M=2 \mathrm{~m} \mathrm{53s}, S D=1 \mathrm{~m} \mathrm{31s})$ than those of EWR $(M=3 \mathrm{~m} \mathrm{54s,} S D=55 \mathrm{~s}, F(1,6)=5.9, p=.051)$, because LGA is situated further away from the departure fixes. This is consistent with the idea that airports situated farthest from the departure fixes tend to pick and get slots before other airports that are situated closer. The departures from airports that are closer to the departure fixes therefore need to wait longer to take off.

\section{Towers - Planner coordination of Scheduled Departure Times}

The coordination interface and mechanism worked well. Most of the time, there were two interactions between the Towers (submitting) and the Planner (accepting). About 59\% of the Towers' proposed SDTs were accepted by the Planner without modification. It was expected that there would be more back and forth coordination between the Towers and the Planner in the Arrival Sensitive Departure scheduling condition than in the Departure Scheduling condition, but that was not the case; there was no difference between the two conditions. In both scheduling conditions, the Tower agreed with the Planner's new SDTs 93\% of the time. There were never more than 4 total interactions. After that, the procedure required the Tower and the Planner to call each other and coordinate verbally.

When not in agreement with the Towers, the Planner moved the SDTs earlier $12 \%$ of the time, and later $28 \%$ of the time. Because of the ongoing Tower SDT requests, the schedules at the departure fixes filled up quickly. Thus there were fewer opportunities for the Planner to propose earlier times than the ones the Tower requested. The Planner also had to move SDTs later when two towers requested the same slot at a departure fix.

Towers competed for the same slot $27.5 \%$ of the time and about half of this competition was between LGA and EWR (14\% of the time). The Planner developed a strategy to handle competing requests. As expected, when the first aircraft's callsign SDT was already accepted (frozen) and the second aircraft's callsign SDT competed for the same time, the Planner would delay the aircraft with the second callsign to a later time $(68-75 \%$ of the time). However, when two aircraft, one from each tower, were competing for the same time, but neither time had been accepted yet (unfrozen), the Planner would tend to accept the SDT of the aircraft callsign with the largest delay (compared to its EDT), and would further delay the other aircraft, as shown in
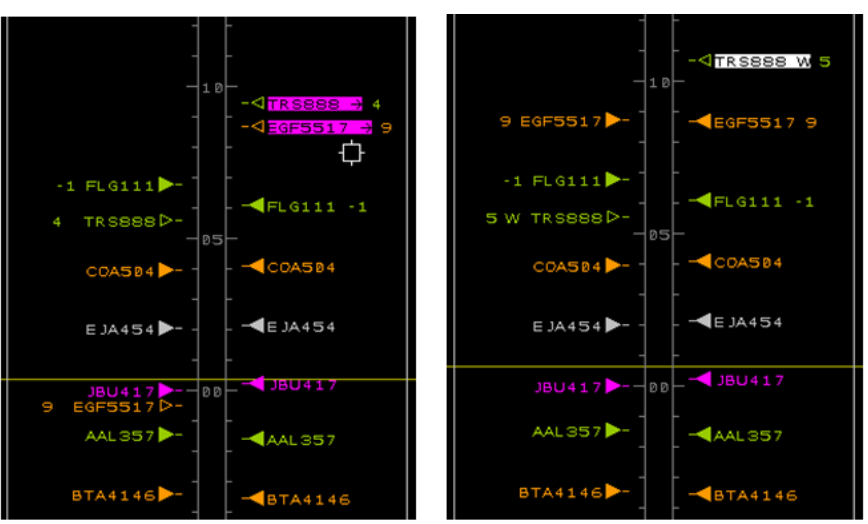

Figure 6. Example of two departures competing for the same STA at the departure fix (in magenta) on the left, and the outcome of the Planner's decision on the right. Fig. 6. In this example, EWR sent a SDT request to the Planner for EGF5517. That proposed scheduled time was 9 minutes later than the EDT. At the same time, LGA sent a SDT request to the Planner for TRS888. The proposed scheduled time was only 5 minutes later than the EDT. In this case, the Planner accepted EWR's request, and moved LGA's time to the next available time (120s later), and proposed the new time to LGA tower, who accepted it. The above example indicates that the Planner did not appear to have followed the "first come, first served" rule. LGA requested slots first $43 \%$ of the time when competing with EWR, but was further delayed $65 \%$ of the time. Similar results were obtained with EWR and TEB. Overall, it seems that the Planner tried not to exacerbate EWR surface delays, by delaying LGA and TEB aircraft more often. Eventually, this strategy may have been judicious, as LGA still ended up with less average surface delays than EWR (probably due to its further distance from the departure fixes). 


\section{Takeoff delays}

We expected more delays at takeoff in the Departure Scheduling condition than in the Arrival Sensitive Departure Scheduling condition, because the SDT may not be feasible due to an arrival's ETA. Despite the surface confederate's attempt to match the departures' SDTs with the EDTs, marginal takeoff delays remained. Figure 7 shows examples of the lack of precision in the scheduling condition without arrivals' STA information being given to the Tower at the scheduling time. The runway timelines in Fig. 7 show ETAs and EDTs on the left side, and SDTs on the right side. Arrival ETAs are in white. On the left timeline, it can be seen that AAL562 and JBU272 SDTs on the right are in front of the arrivals' (DAL432 and AWI533) ETAs on the left. The surface confederate moved AAL562's EDT after the arrivals' ETA (DAL432), and then moved JBU272's

EDT to be 60 seconds after another departure (ASA667), but 60 seconds in front of the next arrival (AWI533). In the right column, the departure EDTs are lined up to take off right after arrivals land. Note that the Towers scheduled departures around the arrival STAs. The confederate who released the departures adjusted the takeoff times based on departure and arrival ETAs.

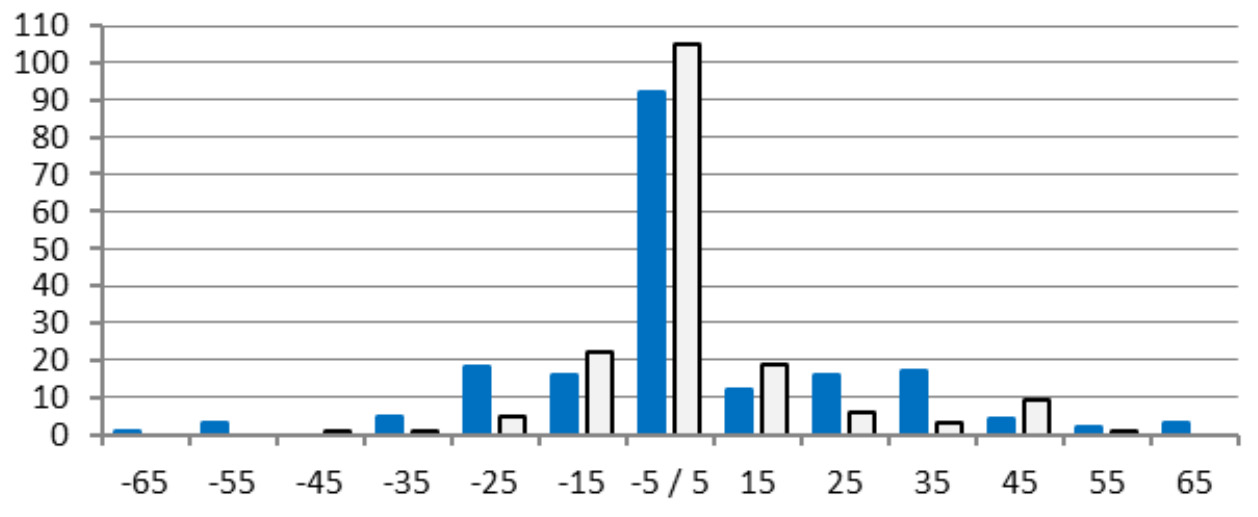

Dep. Scheduling $\quad \square$ Arr. Sensitive Dep. Scheduling

Figure 8. Histograms of takeoff delay by departure scheduling condition.

Positive delays means aircraft took off early, negative delays means aircraft took off late.

Figure 8 shows the distribution of the delays by 10s bins within $65 \mathrm{~s}$ (late) to $65 \mathrm{~s}$ (early). SDT delays at takeoff were computed by deducting actual takeoff times minus scheduled takeoff times. Delays were very similar in means and variances across the two scheduling conditions $(M=-3.4 \mathrm{~s}, S D=16.1 \mathrm{~s}$ in the Arr. Sensitive Dep. Scheduling, vs. $M=-2.1 \mathrm{~s}, S D=20.6 \mathrm{~s}$ in the Dep. Scheduling), $F(1,542)=0.6, p=n s$. The range of the delay was narrower in the Arrival Sensitive Departure Scheduling condition (from 38s early, to $-85 \mathrm{~s}$ late, range 123s) compared to the Departure Scheduling condition (from 99s early to $88 \mathrm{~s}$ late, range 187s). A Levene's test comparing the variances of the two conditions is marginally significant, $F(1,542)=3.3, p=.07$. There were more frequent delays in the -35 to $35 \mathrm{~s}$ range in the Arrival Sensitive condition, whereas the less precise scheduling condition has longer tails and is more widely dispersed around the mean, as would be expected. 
In the No Tools condition, departures took off with more ETAs' being tied at the departure fixes. Figure 9 shows an example of the departure flow over the two departures fixes (BIGGY and WHITE) when the releases of the departures are not scheduled (No Tools condition). Two-way or three-way ties are frequent. In the example, it can be seen that the controllers are creating the desired amount of in-trail spacing as the departures' ETAs are drawing nearer to the departure fixes at the bottom.

\section{B. Controlling and Delivering Departures at Departure Fixes \\ 4. Earlier study}

Prior to this study, we conducted another simulation with the same traffic, airspace, and controllers, and tested whether the CMS$\mathrm{D}$ tools would help controllers deliver departures with better accuracy at departures fixes. ${ }^{24}$ In this study, there was no coordination of the SDTs between the Towers and the Planner. The departures were scheduled and actual takeoff times were scripted to have delays ranging from 90 s late to 90 s early, with the median at $50 \mathrm{~s}$ on each side. The scenarios were run under three experimental conditions: No CMS-D tools, CMS-D tools with single RNAV routes, and CMS-D tools with multiroute options. The results showed that with the same amount of departure fix delay at takeoff
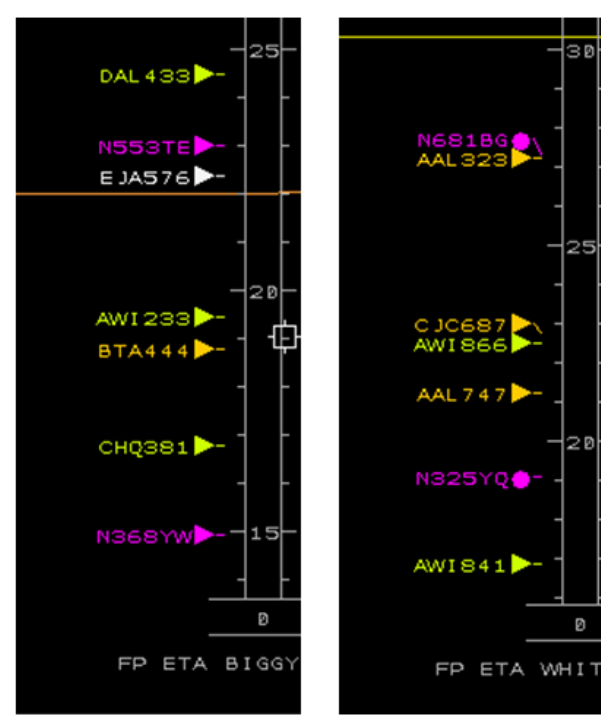

Figure 9. Example of departure ETAs at BIGGY and WHITE in the No Tools condition.

across conditions, controllers delivered aircraft with twice as much precision when they used the CMS-D tools (Single route $M=10.8 \mathrm{~s}, S D=4.5 \mathrm{~s} \&$ Multiroute $M=10.1 \mathrm{~s}, S D=2.8 \mathrm{~s}$ ) than when they did not (No Tools $M=20.7 \mathrm{~s}$, $S D=4.5 \mathrm{~s}), F(2,11)=8.9, p=.007)$. Without CMS-D tools controllers had to vector and delay aircraft to meet the in-trail spacing requirements at the departure fix. With the CMS-D tools, the controllers could take corrective actions as soon as aircraft took off. This was not possible without delay information and slot markers displayed on the scope. The slot markers provided a moving target that helped controllers conform aircraft to their STAs at the departure fixes as the aircraft progressed along its trajectory. The multiroute tool provided some advantages in that it reduced controller coordination and manipulation of aircraft by updating the route. In this earlier study, controller workload was also better distributed between the sectors in the tool conditions. We expected to find similar benefits when controllers used the CMS-D tools when the departures were scheduled.

\section{Controllers' workload}

In the current study, in the No Tool condition with uncoordinated SDTs at the runways, controllers also took more corrective actions to provide the required in-trail spacing near the departure fixes. Figure 10 shows the lateral tracks of the departures to the departure fixes in the three tool conditions. Route deviations due to vectoring appeared more frequently in the No Tools condition than when controllers used the CMS-D tools and the multiroute options. Deviations also seem to take place in the latter portion of the routes. A pattern of fanning tracks can be observed inside both Liberty sectors. 


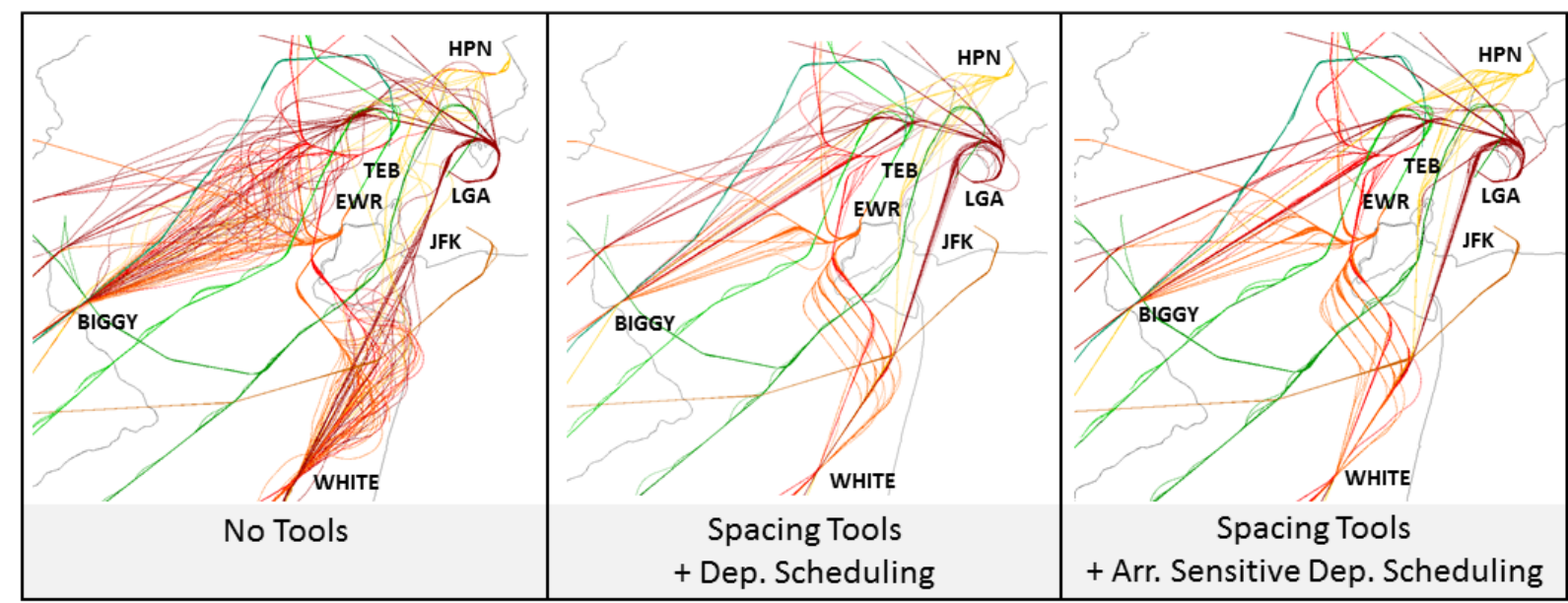

Figure 10. Overlay of departure and arrival tracks in the three tool conditions.

Figure 11 shows the spatial distribution of speed and heading clearances across the sectors. The plot on the left is an example of clearances of a run in the No Tools condition, and shows a large number of heading clearances, compared to the plot on the right of a run when the controllers had the tools. In the No Tools condition run, as can be seen, Liberty South issued many heading clearances inside his airspace, especially to the EWR and TEB departures which he gained control of rather late, compared to the LGA departures. This meant that he often had to vector the EWR and TEB departures to sequence them with sufficient in-trail spacing.

\section{No Tools}

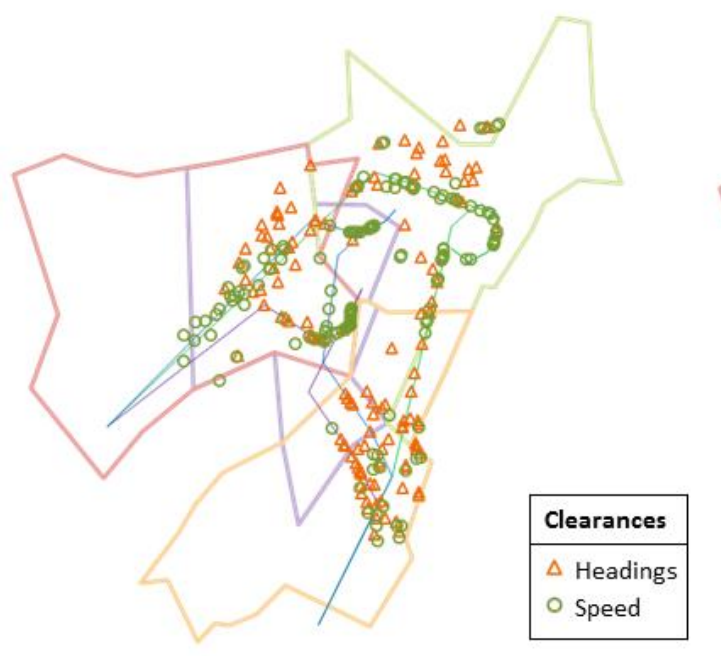

\section{Spacing Tools + Dep. Scheduling}

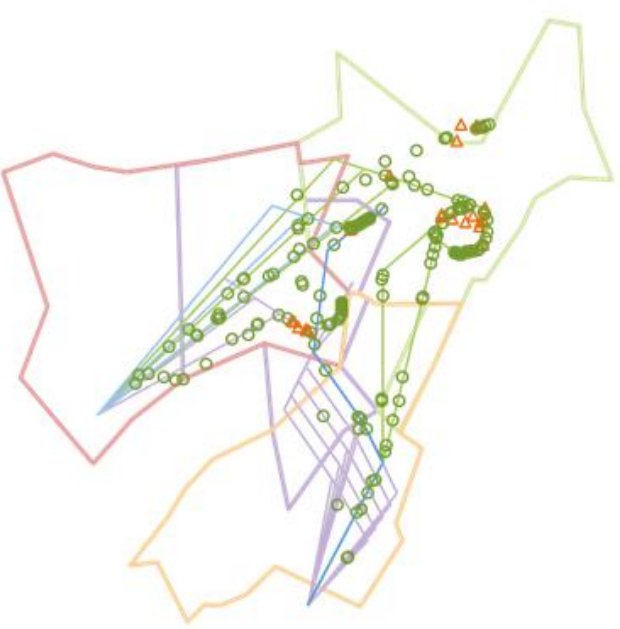

Figure 11. Lat-Long plots of heading and speed clearances.

The plot on the right in Fig. 11 shows a run in a tool condition, with controllers using CMS-D tools and the multiroute options. In this condition, controllers issued significantly fewer heading clearances and issued speed clearances earlier and yet aircraft rarely deviated from the planned trajectories. This suggests that the delay absorption was addressed earlier by the departure controllers. In the No Tools conditions, the departure controllers are oblivious of the most efficient spacing of each aircraft at the departure fix.

Figure 12 shows an example of the Liberty South controller separating and sequencing a 3-way tie between aircraft going to WHITE. Since color-coding was also used on the timelines, a controller could tell that in this example, AWI841, the green aircraft, came from LGA, the purple one, N325YQ, came from TEB, and the orange one, AAL747, came from EWR. Although they all currently were flying at different altitudes, the in-trail restriction required the controller to space and sequence the three aircraft at the fix. In this case, the higher (and thus faster) green aircraft was left on its current heading to cross WHITE first. Next in altitude was the purple aircraft which was 
delayed slightly, and placed second. The orange aircraft was the lowest in altitude, and thus slowest, and was Sturned and placed third.

\section{Controllers' clearances}

As shown in Fig. 13, terminal controllers issued 1.8 times fewer clearances in the two tool conditions $(M=105$, $S D=13 \& M=97, S D=14)$ as in the No Tools condition $(M=$ $189, S D=25), F(2,11)=30.3$, $p=.000$.

Overall, the two Liberty controllers issued 2.3 times more clearances $(M=91, S D=$ 48) than the two departure controllers $(M=39, S D=12)$, $F(2,9)=143.0 \quad p=.000$. However, this is due to an interaction effect between the tool conditions and the sectors. This interaction effect, as shown in Fig. 14, reflects the observations made earlier that the Liberty controllers issued 2.6 times more clearances in the No Tools condition $(M=$ $155, S D=18)$ than in the two tools conditions $(M=62, S D=6 \& M=57, S D=3)$. On the other hand, the two other departure controllers issued about the same number of clearances in the tools conditions $(M=43, S D=6 \& M=$ $39, S D=3)$ as in the No Tool condition $(M=$ $34, S D=18), F(2,9)=61.0 p=.000$. In the No Tools condition, the Liberty controllers issued about 4.5 times more clearances $(M=$ $155, S D=18)$ as the other departure controllers $(M=34, S D=14)$. In the tool conditions, the two Liberty controllers had less work to do to space aircraft because the departure controllers had delay information at their disposal and could take mitigating actions early. The additional work for the departure controllers in the tool conditions was not difficult for them, even though it made a significant difference in lightening the load of the Liberty controllers.

\section{Flight time}

As a result of the uncoordinated release times in the No Tools condition, terminal controllers had to delay aircraft in the air to create the necessary in-trail spacing. The accrued airborne delay can be deduced from the aircraft flight time from the runway to the departure fixes. As shown in Fig. 15, aircraft departures flew on average about 50s longer

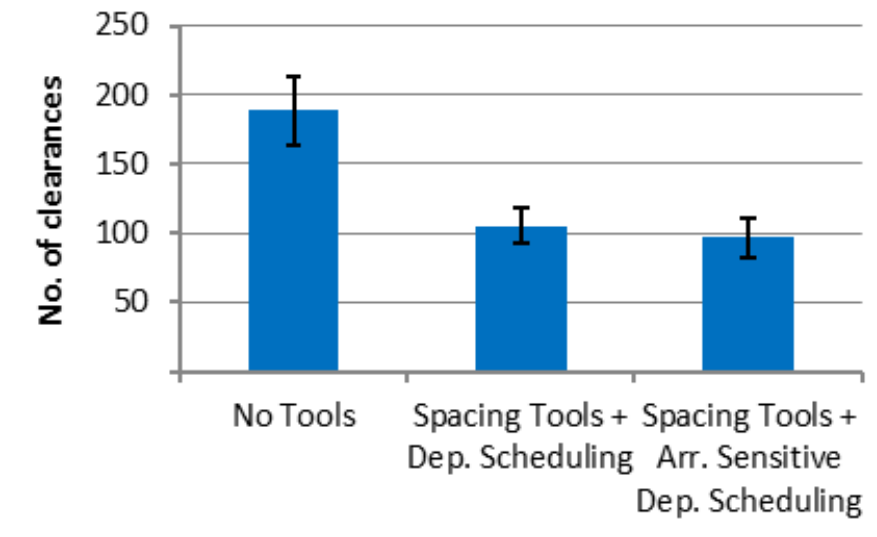

Figure 13. Total number of clearances as a function of tool condition. Error bars $=S D$ s.

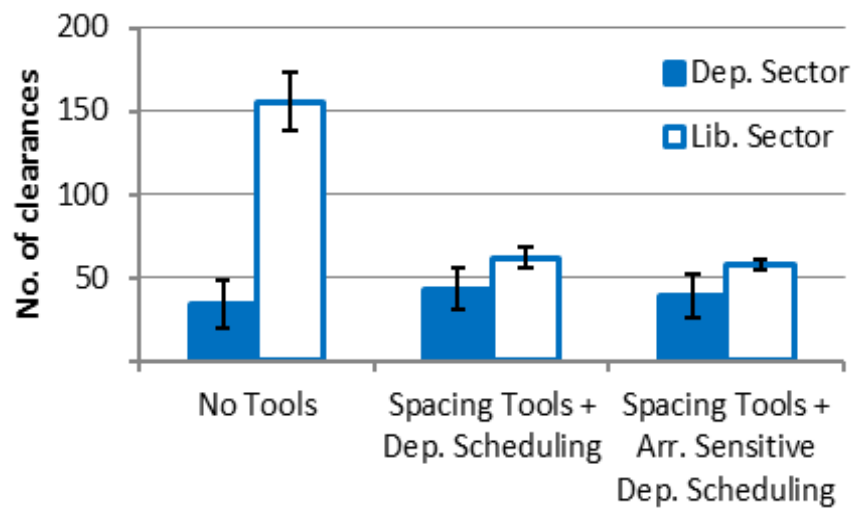

Figure 14. Total number of clearances as a function of tool condition and sector. Error bars $=S D$ s. 
in the No Tools condition $(M=703 \mathrm{~s}, S D=28 \mathrm{~s})$ compared to the Tool conditions $(M=655 \mathrm{~s}, S D$ $=7 \mathrm{~s} \& M=656 \mathrm{~s}, S D=14 \mathrm{~s}), F(2,11)=8.6, p=$ .008 .

\section{Delays at departure fixes}

The above results suggest that controllers were more efficient in managing the flow of departures with the CMS-D tools. Were they more precise with the CMS-D tools in delivering aircraft at the departure fixes? The answer appears to be yes, as shown in Fig. 16 . The delay between the target in-trail spacing and the actual spacing was converted in time in the No Tools condition, and was compared with the absolute value of both early or late delay times of arrival at the departure fixes.

The delays were twice as big in the No Tools condition than values in the scheduling and spacing tool conditions $(M=9.5 \mathrm{~s}, S D=3.7 \mathrm{~s} \&$ $M=8.8 \mathrm{~s}, S D=1.7 \mathrm{~s})(M=21.3 \mathrm{~s}, S D=4.2 \mathrm{~s})$, $F(2,11)=17.4, p=.001)$, as shown in Fig. 16 . This result shows the increased precision attainable using scheduled based tools versus using in-trail distance. Flights crossed the departure fixes at an average ground speed of 385 knots per second. Twenty-one seconds therefore represents an error of $2.2 \mathrm{~nm}$, approximately a $19 \%$ error of the $12 \mathrm{~nm}$ spacing target. On the other hand, 9s of delay represents an $8 \%$ error that was achievable with the spacing tools.

\section{Controllers' ratings}

At the end of each run, controllers filled out surveys on operations, workload, and safety. Ratings were on a Likert scale ranging from 1 to 5 with 5 being the highest.

On the post-run surveys, controllers were asked after each run, "If you vectored, please indicate how frequently it was due to the following requirements: Metering-scheduling or Safety-separation". As can be seen in Fig. 17, it was almost always to meet the in-trail metering and scheduling constraints, and hardly ever for safety or separation purposes, $t(28)=9.2, p=$ 001.

At the end of each run, controllers rated how much mental activity was required during the busiest time, as shown in Fig. 18. On average, controllers rated the No Tool condition as Moderate to High $(M=3.7)$ compared to the Spacing Tools + Dep. Scheduling condition $(M=$ $2.5)$, and to the Spacing Tools + Arr. Sensitive Dep. Scheduling condition $(M=2.8), F(2,9)=$ $5.5, p=.03$.

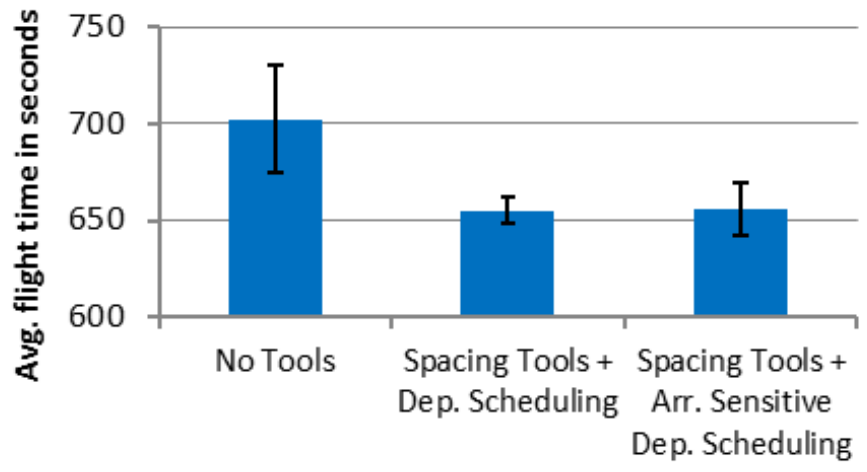

Figure 15. Average flight time in seconds as a function of tool condition. Error bars $=S D$ s.

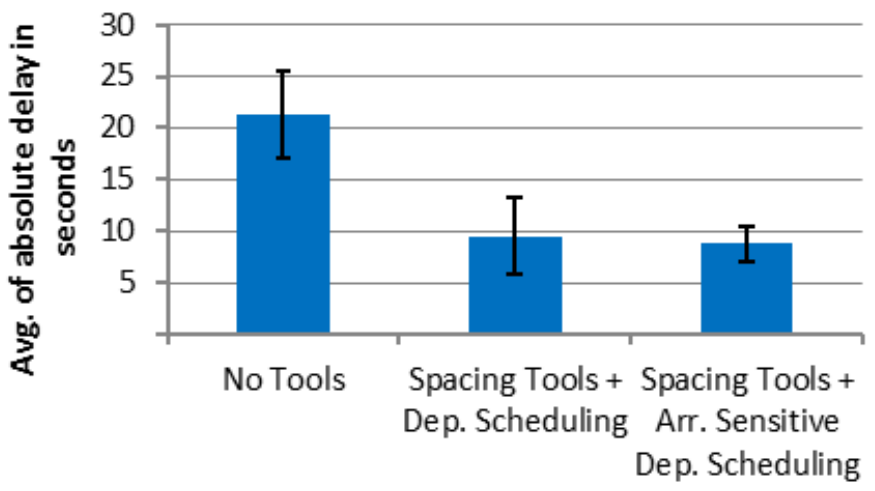

Figure 16. Average absolute delay at the departure fixes as a function of tool condition. Error bars $=S D s$.

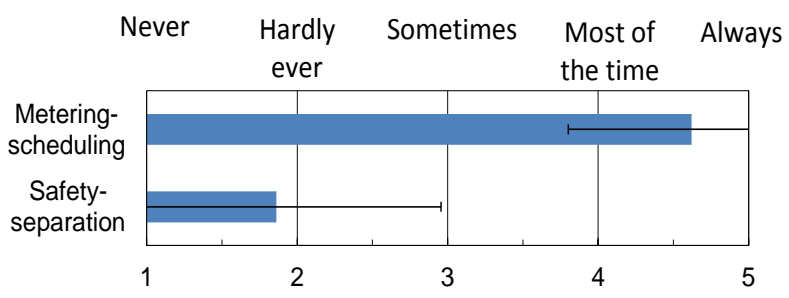

Figure 17. Average frequency of using vectoring for metering/scheduling purpose versus for safety reasons. Error bars $=S D$ s.

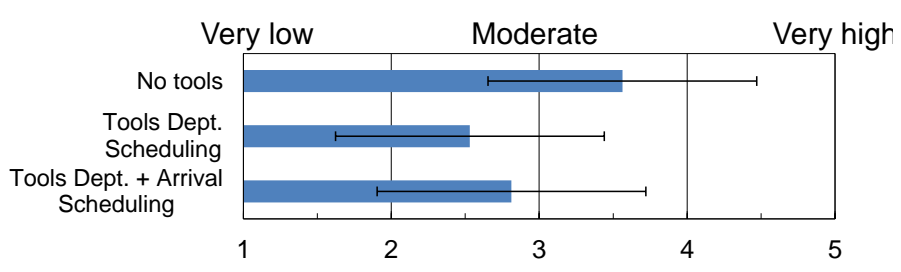

Figure 18. Controllers' ratings of mental activity as a function of tool condition. Error bars $=S D$ s. 
At the end of the study, controllers rated their workload in the different tool conditions. The results can be seen in Fig. 19. Overall, all controllers rated their workload highest in the No Tools condition. The Liberty South controller rated his workload as $5=$ very high, the maximum score, followed by the Liberty West controller, who rated his workload as a $4=$ somewhat high. All other controllers rated their workload as moderate. In contrast, all controllers rated workload as somewhat low (2) in the two tool conditions.

Controllers also rated the acceptability of workload. Not surprisingly, in the No Tools condition, Liberty South rated workload as a 2 on a 1 to 5 scale, i.e., as not very acceptable. The other controllers, even though they had rated workload as moderate or somewhat high in the No Tools condition, found that workload was somewhat acceptable (3), or rated it as a 4, between "Somewhat acceptable" and "Very acceptable". In the tool conditions, all controllers rated workload acceptability as either a 4 or a 5 on the 1 to 5 scale.

As shown in Fig. 20, controllers rated the safety of operations in all the conditions as very close to "5" or "Very acceptable" in both the No Tools condition $(M=4.74)$ and the tool conditions $(M=4.94)$, as shown in

After each run, controllers rated the quality of the flow they received on a 1 to 5 scale with $1=$ Poor, $3=$ Adequate, and $5=$ Very good. As shown in Fig. 21, overall controllers rated that they received better flows in the tool conditions $(M=3.2, M=$ 3.3), than in the No Tools condition $(M=2.3), F(2,8)=7.3, p=.02$.

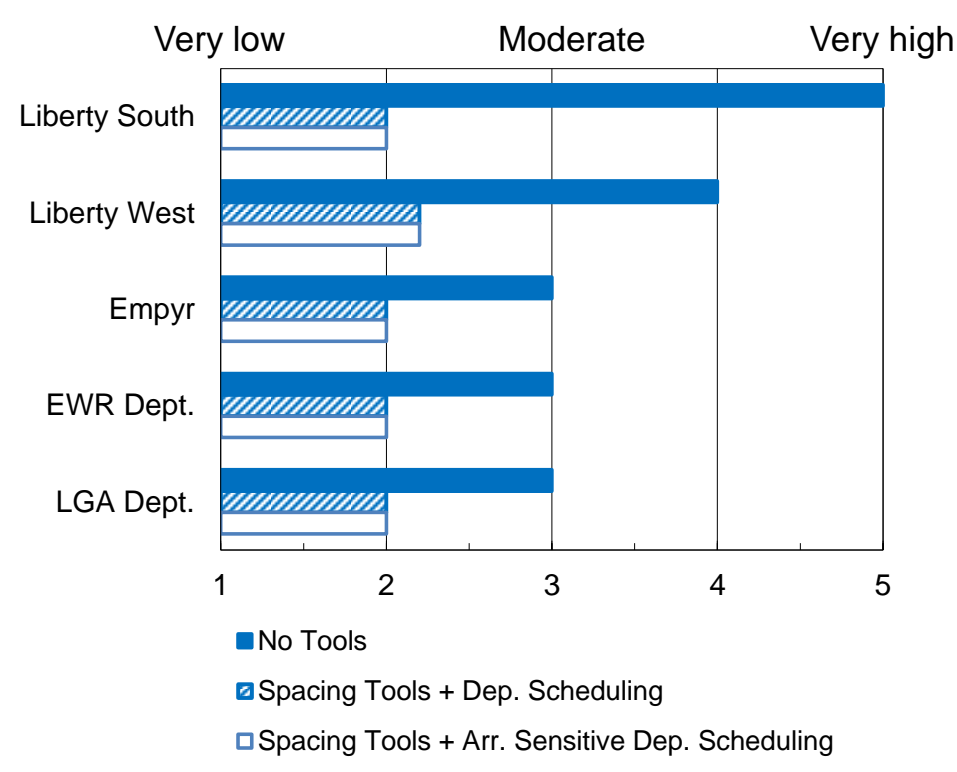

Figure 19. Controllers' ratings of workload as a function of tool condition.

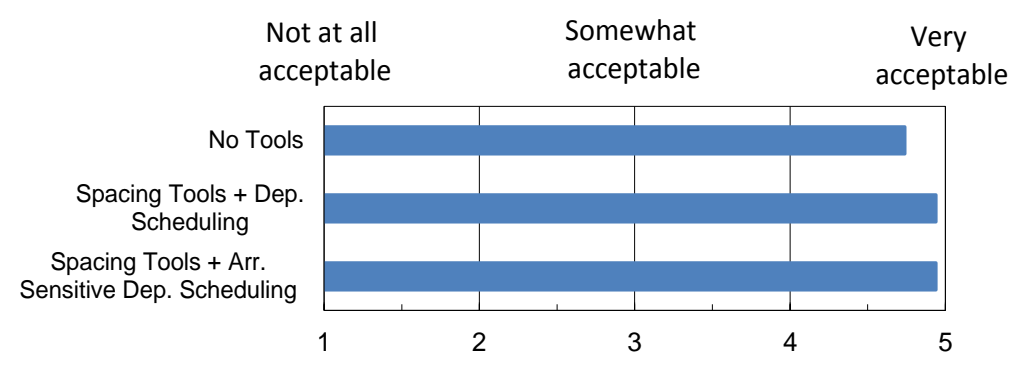

Figure 20. Controllers' ratings of safety as a function of tool condition.

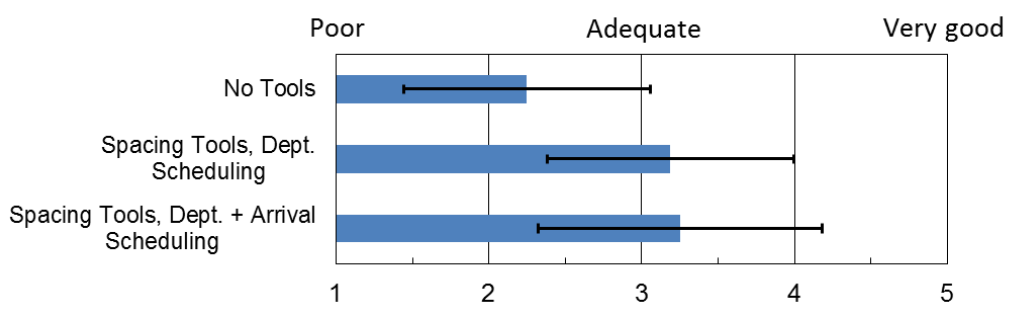

Figure 21. Ratings of quality of flow received as a function of tool condition. Error bars $=S D$ s. 


\section{Exploratory Runs: Using CMS-D without Coordinating Scheduled Takeoff-Times}

At the end of the data collection, we wanted to see how well controllers would do with the CMS-D tools when departure releases were not coordinated between the Towers and the Planner. In this condition, the Towers released departures following the MINIT restrictions set by the Metroplex Planner. The Planner, on the other hand, could see the departure ETAs on the departure fix timeline, as well as floating departure STAs (STAs that were the same as ETAs). The Planner also knew when the departure would get close to its expected departure time (EDT) from the runway timeline. The Planner could then set a schedule by freezing the STAs on the departure-fix timelines. The Planner froze the STAs before the departures took off, trying to make the STAs achievable. As opposed to the tool conditions, the Towers and the Planner did not coordinate the SDTs, nor did the surface confederate try to match the EDT with the SDT. Thus the schedule was established without Tower input and awareness, and the Towers released departures as they would today when meeting the metering constraints. Terminal controllers, on the other hand, were operating with the CMS-D tools.

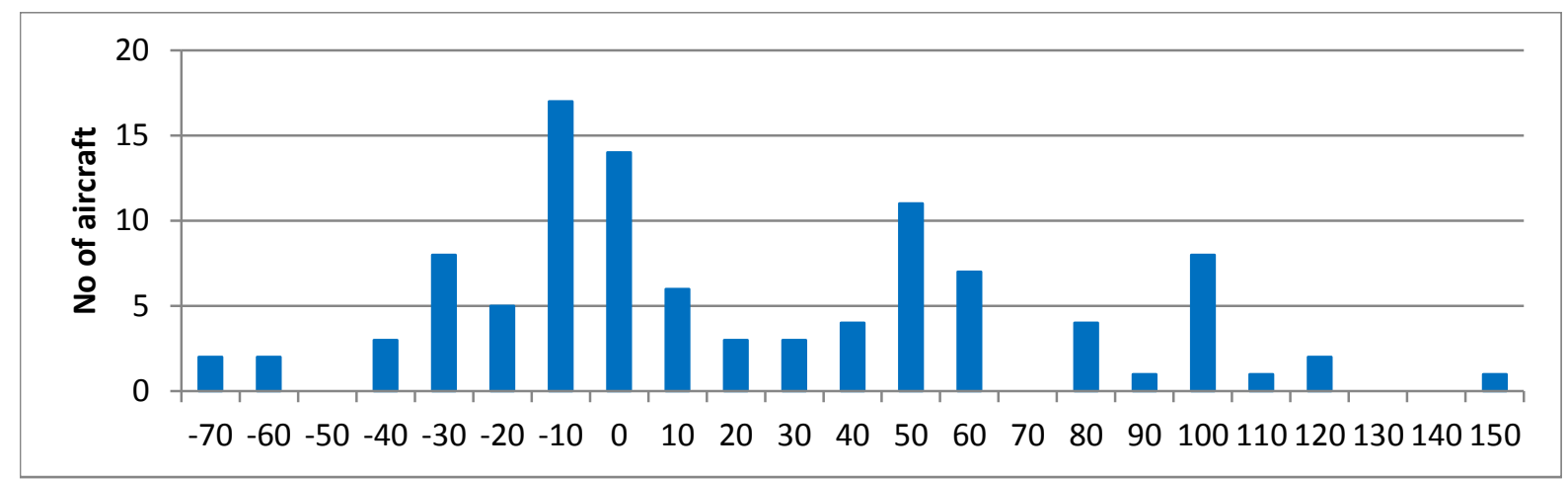

Figure 22. Histograms of takeoff delay in seconds set by the Metroplex Planner during the two exploratory runs. Positive delays indicate aircraft took off early, negative delays indicate aircraft took off late.

The Planner set the takeoff times for the departures to reflect his assessment that it was easier to delay than to speed up an aircraft to meet its scheduled time, as shown in Fig. 22. In this figure, the positive values indicate that departures took off earlier than their STAs. The negative values indicate that departures took off later than their STAs. The above distribution shows 1) that the Planner tried to avoid creating negative delays, where the aircraft's ETA was behind its STA. Instead, he tried to move STAs to a later time than the ETAs (so that the aircraft was early). There was only up to a minute of negative delay to be made up (Max =71s), and a wider tail of positive delays or earlier takeoffs (Max $=-149 \mathrm{~s})$. Delay values in general tended to be early $(M=23 \mathrm{~s}, S D=45 \mathrm{~s})$. The Planner participant reported that, in his opinion, only a minute of negative (late) delay was manageable for the controllers. He thought that larger late delays would require unrealistic speeds and shortcuts to meet the STAs. On the other hand, he thought that delaying an aircraft that was too early was easier to accomplish, since the CMS-D tools would inform the controller how much to slow the aircraft. His strategy allowed controllers to absorb delays without losing throughput.

As shown in Fig. 23, with the CMS-D tools alone (far right column), controllers were able to deliver aircraft at the departure fixes with the same precision that was achieved with the CMS-D tools plus the scheduling tools. The absolute delay values with just the CMS-D tools $(M=10.5 \mathrm{~s}, S D=$ $0.7 \mathrm{~s})$ were nearly identical to those with the CMS-D and the scheduling tools $(M=9.5 \mathrm{~s}$, $S D=3.7 \mathrm{~s}, M=8.8 \mathrm{~s}, S D=1.7 \mathrm{~s}$, , both of which were half the delay values in the No Tools condition $(M=21.3 \mathrm{~s}, S D=4.2 \mathrm{~s})$, $F(2,13)=13.3, p=.001)$.

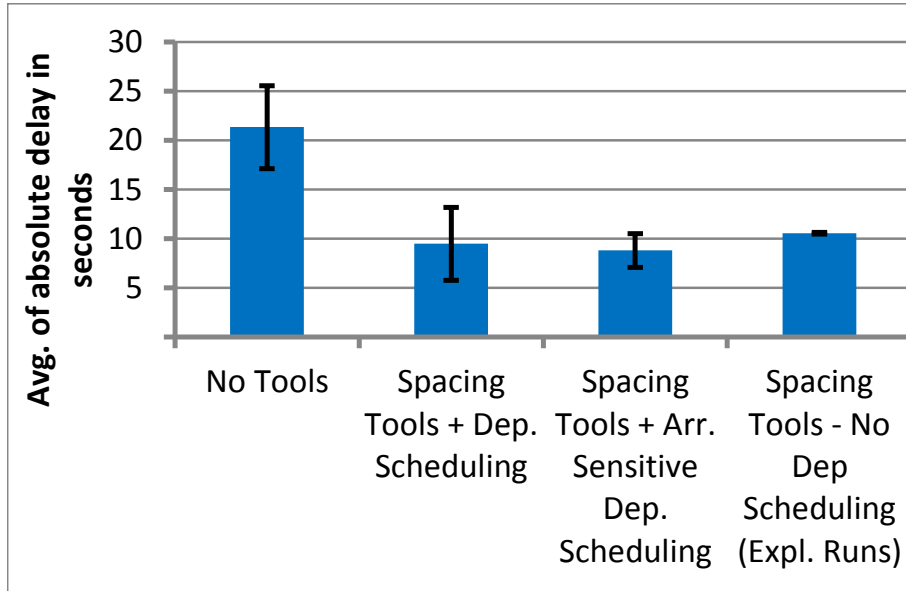

Figure 23. Average of absolute delay at the departure fixes as a function of tool condition. Error bars $=S D$.

American Institute of Aeronautics and Astronautics 


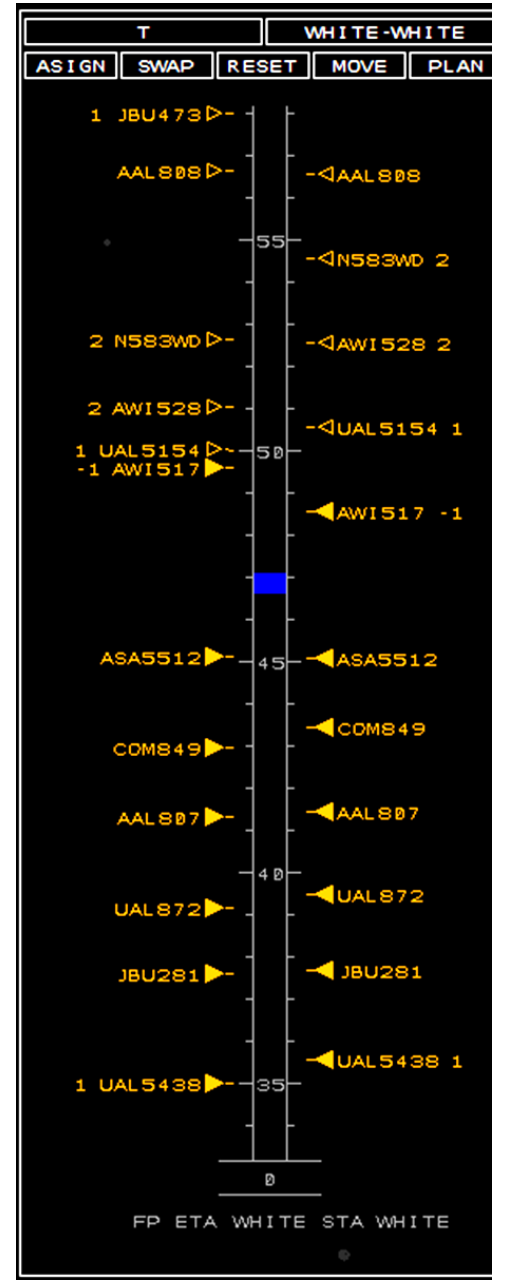

Figure 24. Example of departure delays at WHITE departure fix during the exploratory runs.
Figure 24 shows an example of the delays of departures flying to WHITE during an exploratory run. It can be seen that delays are absorbed somewhere around 10 minutes before the departure fix (at the bottom of Fig. 24) inside the freeze horizon. STAs froze when departures were about to take off (around 15 minutes before the departure fix). As can be seen, delays were notably higher on the runway before the freeze horizon.

At the end of each CMS-D exploratory run, controllers rated how much mental activity was required during the busiest time. As shown in Fig. 25, all controllers, except EWR Departure, rated the mental activity as higher in the exploratory runs $(M=3.4)$ than they did in the CMS-D plus scheduling condition $(M=2.5, F(1,4)=7.0, p=.057$, marginally significant.

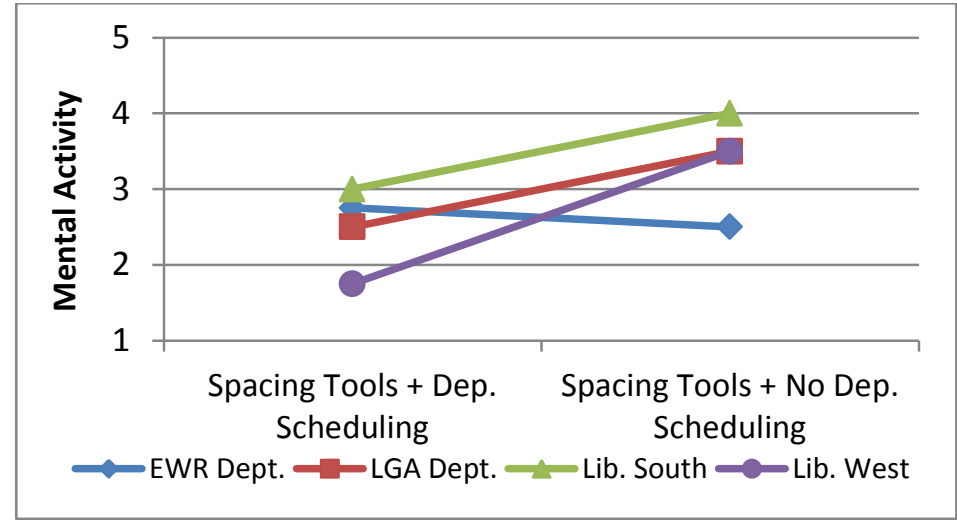

Figure 25. Average mental activity ratings during the exploratory runs (right) compared with ratings during the departure scheduling condition (left).

\section{Discussion}

In this paper, we presented a concept of time-based scheduling and delivering departure aircraft to the terminal boundary of a metroplex. Our goal was to improve the problematic management of departure flows into the en route airspace, as well as to improve scheduling departures from multiple airports. Our concept addresses current challenges such as schedule management in a metroplex environment, coordination of departure release times, and in particular, flight time compliance. We evaluated the concept in a HITL that emulated the NY metroplex airspace, where aircraft from three airports were tactically scheduled and released to fly through two departure fixes with flow restrictions. Overall, the results show that, when the concept was simulated, there was an improvement in predictability, controllability, and precision of operations.

\section{Main Findings}

The TMCs from the towers and the Metroplex Planner successfully coordinated Scheduled Departure Times off the runway to the departure fixes. Fifty-nine percent of the time, the towers' SDTs were accepted by the Planner without modifications. Twenty-eight percent of the time, two airports would compete for an identical slot at the departure fix. If the Planner had not yet accepted a request, he would favor the departure that already had the most taxi-out delay, and give the later slot to the departure that had the least taxi-out delay. Taxi-out delays were increased by a minute (40\%) when towers released departures following MINIT restrictions in the No Tools condition, compared to when there were CMS-D tools and towers coordinated SDTs with the Planner $(M=3 \mathrm{~m} 30 \mathrm{~s})$, showing the advantage of scheduling based on departure fix demand rather than the constant restriction of MINIT. LGA had 35\% less taxi-out delay compared to EWR, because LGA had longer flight times to the departure fixes, and the LGA Tower TMC could request SDTs before the EWR Tower TMC could.

Once scheduled, the departures did not always take off on time due to runway constraints. The range of takeoff delay was smaller when the Tower TMCs knew when the arrivals were scheduled at the runway, so they could 
schedule departures accordingly. When the Tower TMCs were oblivious of the arrival STAs, there was a slightly higher variance and a larger range of delay times from the scheduled times that the departures reached the departure fixes. The Minutes-in-Trail release of departures across the metroplex in the No Tools condition produced a traffic demand that was uncoordinated at the departure fixes, with many departures arriving at the same time. This caused controllers near the fixes to delay aircraft and vector them to obtain the required in-trail spacing, thus increasing workload and trajectory inefficiencies.

Without the CMS-D tools, the departure controllers were unaware of the predicted spacing of aircraft at the departure fixes. It was only when departures entered the Liberty sectors that control the departure fixes, that corrective actions could be taken to space aircraft properly. For this reason, Liberty controllers issued four times as many clearances in the No Tools condition compared to the tool conditions. In the No Tool condition, lateral track plots show frequent vectoring particularly in the Liberty sectors. To manage the in-trail spacing, controllers delayed departures in the air by an average of 50 seconds in the No Tool condition compared to the tool/scheduling conditions.

Controllers delivered departures with twice as much precision in the tool conditions (10s, $8 \%$ error) than without the tools (21s, 19\% error). The CMS-D tools helped controllers condition aircraft early across all sectors. This was possible because the departure controllers were given delay information from the tools as soon as the aircraft took off. This information was available from the early/late indicator on the datablock and also from the prototype timelines showing the departure fix estimated time of arrival (ETA) compared to its scheduled time (STA). But perhaps most important, the slot markers showed the visual location of the scheduled position of the aircraft (STA) so that the controllers could compare it with where the aircraft actually was (ETA). Knowing this information early helped distribute the absorption of delay across sectors and enabled the controllers to monitor the delay of the aircraft as it moved along its entire trajectory. The initial prediction of a departure's ETA can change with the various uncertainties such as climb rate and takeoff delay. In the tool conditions, the departure controllers were able to deal with any changes immediately, relieving the Liberty controllers near the fix from later scrambling to separate aircraft. In effect, the CMS-D tools helped controllers to assess the compliance of aircraft to its STA at any given time. In the survey responses, controllers indicated that workload was much more evenly distributed in the tool conditions and also rated the level of workload as more acceptable. They rated operations as being safe in all conditions.

In two exploratory runs, departures were released without scheduling coordination, following the MINIT spacing restrictions. However, the Planner set STAs for the departures at the departure fix timeline prior to aircraft takeoff and controllers were given the CMS-D tools to space and sequence departures according to the MIT restrictions. The Planner set STAs to a later time than ETAs when possible. By so doing, he relieved traffic demand pressure and provided controllers with more manageable delays. Throughput was not affected. His strategy supports the notion that controllers can more easily delay aircraft than make up time. This strategy seemed successful. Controllers achieved the same level of precision at the departure fixes as when the departures were scheduled by the Towers to available slots at the departure fixes, but with a slightly higher workload.

The exploratory runs showed that the CMS-D tools helped the controllers deliver aircraft within 10 seconds of their STAs at the departure fixes, regardless of the extent of initial takeoff delays. This reflects the findings of the earlier simulation, where takeoff delays were scripted to range from -90s to 90s at takeoff, but the CMS-D tools still provided large benefits. ${ }^{24}$ It appears that the CMS-D tools, with or without multiroute options, would provide resilience and add robustness to any scheduling system. Such resilience would mitigate uncertainties inherent in departure climb rates and takeoff times. Of course, the less precise the takeoff time, the higher the delay for the controllers to make up, thus the harder the controllers have to work to meet the time at the departure fix. If vectoring is needed, inefficient trajectories result. Hence, there are advantages to scheduling and having aircraft meet those scheduled times at the departure fixes. In sum, scheduling departures tactically and using conformance tools such as the CMS-D tools may be an efficient approach both for the controllers and for the airlines.

\section{E. Need for Further Investigation.}

Further research is needed to understand the separate benefits of the scheduling and the CMS-D tools.

Also, more research is needed to create an optimal schedule at the center boundary to manage both the volume of departures and the integration of departures into overhead flows. In this study, the schedule at the terminal boundary matched the en route restriction imposed there. The terminal controllers meeting the STAs thus met the needs of the en route sector. However, in reality, BIGGY and WHITE departures fixes have different types of constraints. The flow to BIGGY needs to be merged with an overhead stream in the en route sector. In this case, the departure demand needs to be spaced out enough to fit in the traffic. The flow to WHITE is subject to volume capacity constraints. There is no need to merge traffic until the next en route sector. Since applying a fixed MIT restriction 
for en route does not take into account the fluctuation of the terminal demand, the challenge is to couple the departure schedule with a downstream schedule. This would provide more control over the delays and reduce unnecessary restrictions. In the current study there was an excess demand over capacity at the departure fix. However there may be other situations where there may be excess capacity, thus resulting in slack in the schedule. In this case, constraining an aircraft to a specific STA may not be necessary. If there is enough spacing between departures, the STA could be the same as the ETA. When the restriction is meant to control volume, or the rate of demand, then relative spacing may be more important to control than complying to STAs.

More research is needed to assess the impact of using TBFM and scheduling without RNAV routes, and without the support of CMS-D tools, particularly in a stream-insertion scenario. Further research is needed to assess the level of compliance at takeoff and the airborne precision that provide a flow that can fit in the overhead stream without loss of slots/capacity. At the same time, there is also a need to better predict departure fix ETAs so that controllers can provide more precise departures to scheduled points. Finally, there is a need to integrate surface and airborne scheduling solutions to optimize flow management.

\section{Conclusion}

A concept of departure scheduling and delivery at terminal departure fixes in a NY metroplex airspace was successfully tested during a one-week HITL simulation. The scheduling and CMS-D tools provided the necessary information for controllers to achieve a high level of conformance to the schedule at the departure fixes. Further research is needed to understand the benefits of each.

\section{Acknowledgments}

The authors thank Shannon Zelinski and the Airspace System Program at NASA Ames Research Center for supporting this study, as well as the research and the development teams at the Airspace Operations Laboratory who made the outcome possible. Furthermore, the authors thank the controllers who provided invaluable feedback before and after the studies.

\section{References}

${ }^{1}$ Federal Aviation Administration, NextGen Implementation Plan 2014. Washington, DC, 2014.

${ }^{2}$ Prevot, T., Baxley, B. T., Callantine, T. J., Johnson, W., Quon, L. K., Robinson, J. E., and Swenson, H. N. "NASA's ATM Technology Demonstration-1: Transitioning Fuel Efficient, High Throughput Arrival Operations from Simulation to Reality," International Conference on Human-Computer Interaction in Aerospace (HCI-Aero), 2012.

${ }^{3}$ Callantine, T. J., Hunt, S. M., and Prevot, T., "Simulation Evaluation of Controller-Managed Spacing Tools under Realistic Operational Conditions." International Conference on Human-Computer Interaction in Aerospace (HCI-Aero), 2014.

${ }^{4}$ Swenson, H. N., Thipphavong, J., Sadovsky, A., Chen, L., Sullivan, C., and Martin, L., "Design and evaluation of the terminal area precision scheduling and spacing system," Ninth USA/Europe Air Traffic Management Research and Development Seminar (ATM), 2011.

${ }^{5}$ Engelland, S. A., Capps, R. A., Day, K. B., Kistler, M. S., Gaither, F., and Juro, G., "Precision Departure Release Capability (PDRC) Final Report.” NASA/TM-2013-216533, 2013.

${ }^{5}$ Engelland, S. A., and Capps, R. "Trajectory-Based Takeoff Time Predictions Applied to Tactical Departure Scheduling: Concept Description, System Design, and Initial Observations," Eleventh AIAA Aviation Technology, Integration and Operations Conference (ATIO), 2011.

${ }^{7}$ Capps, R. A. and Engelland, S.A., "Characterization of Tactical Departure Scheduling in the National Airspace System," Eleventth AIAA Aviation Technology, Integration, and Operations Conference (ATIO), Virginia Beach, VA, 2011.

${ }^{8}$ Rhodes, L. S. and Rhodes, L. R., Operational Characteristics and Application of Miles-In-Trail Restrictions, The MITRE Corporation, McLean, VA, 2001.

${ }^{9}$ Sheth, K., Gutierrez-Nolasco, S., and Petersen, J. "Analysis and Modeling of Miles-in-Trail Restrictions in the National Airspace System," AIAA 13 ${ }^{\text {th }}$ Aviation Technology, Integration, and Operations Conference, 2013.

${ }^{10}$ Stein, B., Ceniccola, D., and Vincent, D., "TBFM Coupled Scheduling," Fifty-Sixth Annual ATCA Conference and Exposition, National Harbor, MD, 2011.

${ }^{11}$ Capps, R. A., Day, K., Walenciak, E., and Engelland, S. A., "Impact of Departure Prediction Uncertainty on Tactical Departure Scheduling System Performance," Twelfth AIAA Aviation Technology, Integration, and Operations Conference (ATIO), 2012.

${ }^{12}$ Jung, Y. C., Isaacson, D. "Development of Conflict Free, Unrestricted Climbs for a terminal Area Departure Tool," Third AIAA Annual Aviation Technology, Integration and Operations Conference (ATIO), 2003.

${ }^{13}$ Jung, Y. C., and Isaacson, D. R., "Design Concept and Development Plan of the Expedite Departure Path," AIAA Aircraft Technology, Integration, and Operations Forum (ATIO), 2002.

${ }^{14}$ Wang, J. J., Chang, P., and Datta, K., "Life-Cycle Cost/Benefit Assessment of Expedite Departure Path (EDP)," Prepared for NASA Ames Research Center, NASA/CR-2005-212836, 2003. 
${ }^{15}$ Engelland, S. A., Capps, R. A., and Kevin Brian Day. "Precision Departure Release Capability (PDRC) Concept of Operations," NASA/TM-2013-216534, 2013.

${ }^{16}$ Jung, Y. C., Malik, W., Tobias, L., Gupta, G., Hoang, T., and Hayashi, M., "Performance Evaluation of SARDA: An Individual Aircraft-based Advisory Concept for Surface Management," Air Traffic Control Quarterly, Vol. 22, No. 3, 2015, pp. 195-221.

${ }^{17}$ Hayashi, M., Hoang, T. Jung, Y. C., Gupta, G., Malik, W., and Dulchinos, V. "Usability Evaluation of Spot and Runway Departure Advisor (SARDA) Concept in Dallas/Fort Worth Airport Tower Simulation," Tenth USA/Europe Air Traffic Management Research and Development Seminar (ATM), 2013.

${ }^{18}$ Kopardekar, P., Green, S., Brinkman, C., Thompson, P., Evans, M., and Davis, D., "Making Internal Departure Release Operations More Efficient," Fourth AIAA Aircraft Technology, Integration, and Operations Forum (ATIO), 2004.

${ }^{20}$ Doble, N., Timmerman, J., Carniol, T., Klopfenstein, M., Tanino, M., and Sud, V., "Linking Traffic Management to the Airport Surface: Departure Flow Management and Beyond," Eighth USA/Europe Air Traffic Management Research and Development Seminar (ATM), 2009.

${ }^{20}$ Hightower, K., "Integrated arrival and departure capability," Integrated Communications, Navigation and Surveillance Conference (ICNS), Institute of Electrical and Electronics Engineers, Piscataway, 2013, pp. 1-5.

${ }^{21}$ Capps, R. A., Kistler, M. S., and Engelland, S. A., "Design Characteristics of a Terminal Departure Scheduler," AIAA $14^{\text {th }}$ Aviation Technology, Integration, and Operations Conference (ATIO), 2014.

${ }^{22}$ Prevot, T., Smith, N. S., and Palmer, E. A., "The Airspace Operations Laboratory (AOL) at NASA Ames Research Center," AIAA Modeling and Simulation Technologies Conference, 2006.

${ }^{23}$ Prevot, T., Smith, N. S., Palmer, E. A., Callantine, T. J., Lee, P., Mercer, J., Homola, J., Martin, L, Brasil, C., and

Cabrall, C. "An Overview of Current Capabilities and Research Activities in the Airspace Operations Laboratory at NASA Ames Research Center," AIAA 14 th Aviation Technology, Integration, and Operations Conference, 2014.

${ }^{24}$ Kraut, J. M., Chevalley, E., Bienert, N., Omar, F., Borade, A., Parke, B., Yoo, H. S., Palmer, E. A. "Effect of schedule conformance tools for delivering terminal aircraft to departure fixes on task and workload distribution among adjacent sectors," Paper submitted to the Sixth International Conference on Applied Human Factors and Ergonomics, 2015. 